Journal for

.... ImmunoTherapy of Cancer

\title{
Bispecific antibody-mediated redirection of NKG2D-CAR natural killer cells facilitates dual targeting and enhances antitumor activity
}

\author{
Congcong Zhang, ${ }^{1,2,3,4}$ Jasmin Röder, ${ }^{1,4}$ Anne Scherer, ${ }^{1}$ Malena Bodden, ${ }^{1}$ \\ Jordi Pfeifer Serrahima, ${ }^{1}$ Anita Bhatti, ${ }^{1}$ Anja Waldmann, ${ }^{1}$ Nina Müller, ${ }^{1}$ \\ Pranav Oberoi, ${ }^{1,2,3}$ Winfried S Wels (1) ${ }^{1,2,3,4}$
}

To cite: Zhang C, Röder J, Scherer A, et al. Bispecific antibody-mediated redirection of NKG2D-CAR natural killer cells facilitates dual targeting and enhances antitumor activity. Journal for ImmunoTherapy of Cancer 2021;9:e002980. doi:10.1136/jitc-2021-002980

- Additional supplemental material is published online only. To view, please visit the journal online (http://dx.doi.org/10. 1136/jitc-2021-002980).

Accepted 16 September 2021

\section{Check for updates}

(c) Author(s) (or their employer(s)) 2021. Re-use permitted under CC BY-NC. No commercial re-use. See rights and permissions. Published by BMJ.

${ }^{1}$ Georg-Speyer-Haus, Institute for Tumor Biology and Experimental Therapy, Frankfurt, Germany

${ }^{2}$ German Cancer Consortium (DKTK), partner site Frankfurt/ Mainz, Frankfurt, Germany ${ }^{3}$ German Cancer Research Center (DKFZ), Heidelberg, Germany

${ }^{4}$ Frankfurt Cancer Institute, Goethe University, Frankfurt, Germany

Correspondence to

Professor Winfried S Wels; wels@gsh.uni-frankfurt.de

\section{ABSTRACT}

Background Natural killer group 2D (NKG2D) is an activating receptor of natural killer (NK) cells and other lymphocytes that mediates lysis of malignant cells through recognition of stress-induced ligands such as MICA and MICB. Such ligands are broadly expressed by cancer cells of various origins and serve as targets for adoptive immunotherapy with effector cells endogenously expressing NKG2D or carrying an NKG2D-based chimeric antigen receptor (CAR). However, shedding or downregulation of NKG2D ligands (NKG2DL) can prevent NKG2D activation, resulting in escape of cancer cells from NKG2D-dependent immune surveillance.

Methods To enable tumor-specific targeting of NKG2Dexpressing effector cells independent of membraneanchored NKG2DLs, we generated a homodimeric recombinant antibody which harbors an $\mathrm{N}$-terminal single-chain fragment variable (scFv) antibody domain for binding to NKG2D, linked via a human $\lg _{4}$ Fc region to a second $\mathrm{C}$-terminal scFv antibody domain for recognition of the tumor-associated antigen ErbB2 (HER2). The ability of this molecule, termed NKAB-ErbB2, to redirect NKG2Dexpressing effector cells to ErbB2-positive tumor cells of different origins was investigated using peripheral blood mononuclear cells, ex vivo expanded NK cells, and NK and T cells engineered with an NKG2D-based chimeric receptor.

Results On its own, bispecific NKAB-ErbB2 increased lysis of ErbB2-positive breast carcinoma cells by peripheral blood-derived NK cells endogenously expressing NKG2D more effectively than an ErbB2-specific lgG miniantibody able to induce antibody-dependent cell-mediated cytotoxicity via activation of CD16. Furthermore, NKABErbB2 synergized with NK-92 cells or primary T cells engineered to express an NKG2D-CD3 $\zeta$ chimeric antigen receptor (NKAR), leading to targeted cell killing and greatly enhanced antitumor activity, which remained unaffected by soluble MICA known as an inhibitor of NKG2Dmediated natural cytotoxicity. In an immunocompetent mouse glioblastoma model mimicking low or absent NKG2DL expression, the combination of NKAR-NK-92 cells and NKAB-ErbB2 effectively suppressed outgrowth of ErbB2-positive tumors, resulting in treatment-induced endogenous antitumor immunity and cures in the majority of animals.
Conclusions Our results demonstrate that combining an NKAB antibody with effector cells expressing an activating NKAR receptor represents a powerful and versatile approach to simultaneously enhance tumor antigen-specific as well as NKG2D-CAR and natural NKG2D-mediated cytotoxicity, which may be particularly useful to target tumors with heterogeneous target antigen expression.

\section{BACKGROUND}

Immunotherapy with chimeric antigen receptor (CAR)-engineered $\mathrm{T}$ cells has demonstrated remarkable clinical efficacy in patients with B-cell malignancies. ${ }^{1}$ However, successful targeting of more prevalent solid tumors with CAR-T cells still remains a challenge, owing in part to an immunosuppressive tumor microenvironment, limited persistence of infused effector cells, and heterogeneity of target antigen expression. ${ }^{23}$ With respect to the latter, current CAR effector cells typically recognize a single tumor-associated surface antigen, resulting in only limited cell killing in tumors with uneven distribution of the target, and favoring outgrowth of subclones with low or absent antigen expression. ${ }^{4-6}$ Hence, different strategies are being followed to enable recognition of multiple target antigens, which include a combination of different monospecific CAR-T cells and coexpression of two CARs in the same T cells, ${ }^{7}$ or the more complex generation of CARs harboring more than one cell-binding domain. ${ }^{8}$

Another approach harnesses the broad tumor specificity of the natural killer group 2D (NKG2D) molecule in a CAR design, linking the extracellular ligand-binding domain of NKG2D to a heterologous signaling molecule such as CD3ל. ${ }^{9-11}$ NKG2D is an activating receptor expressed by all natural killer 
(NK) cells, $\mathrm{CD}^{+} \mathrm{T}$ cells, and most natural killer T cells, as well as subpopulations of $\mathrm{CD} 4^{+} \mathrm{T}$ cells and $\gamma \delta \mathrm{T}$ cells. ${ }^{11-13}$ NKG2D has multiple membrane-anchored ligands, which in humans include the MHC class I-related molecules MICA and MICB and six UL16 binding proteins (ULBP16). ${ }^{1415}$ These ligands are selectively upregulated by transformed and stressed cells and are widely expressed in almost all cancer types, including lung, breast, kidney, pancreatic, ovarian and prostate cancer, melanoma, leukemia, and glioblastoma. ${ }^{16}{ }^{17}$ Hence, NKG2D ligands (NKG2DLs) are promising targets for adoptive immunotherapy with cytotoxic lymphocytes that naturally express NKG2D or are engineered to express NKG2D-based CARs. ${ }^{9-11} 18$ General safety of NKG2D-CAR T cells has recently been demonstrated in a phase I trial in patients with acute myeloid leukemia/myelodysplastic syndrome or multiple myeloma. ${ }^{19}$ Nevertheless, activity of such NKG2D-CAR cells may be reduced against leukemic stem cells with low or absent expression of NKG2DLs. ${ }^{20}$ Natural NKG2D-mediated immune surveillance can be counteracted through a variety of mechanisms, including downregulation of NKG2DLs on exposure of cancer cells to interferon gamma (IFN- $\gamma$ ) or transforming growth factor beta(TGF- $\beta$ ). ${ }^{21}{ }^{22}$ Tumor cells can also decrease NKG2DL density by proteolytic shedding. ${ }^{23}$ Accordingly, in the sera of patients with cancer, elevated levels of soluble NKG2DLs have been found, in many cases correlating with disease stage and metastasis. ${ }^{12}{ }^{17}$ High concentrations of shed NKG2DLs can act as a competitor and block the interaction of NKG2D with tumor cells, induce NKG2D internalization and degradation, and desensitize immune effector cells. ${ }^{123-25}$

To limit interference by soluble NKG2DLs and redirect NKG2D expressing immune cells specifically to cancer cells of solid tumor origins, here, we designed a bispecific antibody combining two NKG2D-binding and two ErbB2 (HER2)-specific single-chain fragment variable $(\mathrm{scFv})$ domains, linked by an $\operatorname{IgG}_{4}$ Fc region in a single tetravalent molecule. Specific targeting by this NKAB-ErbB2 antibody to antigen-positive tumor cells and enhancement of NKG2D-mediated cytotoxicity was evaluated in in vitro cell killing experiments with primary lymphocytes endogenously expressing NKG2D and established NK-92 and primary $\mathrm{T}$ cells which carry an NKG2D-based chimeric antigen receptor (NKAR) encompassing the extracellular domain of NKG2D fused to transmembrane and intracellular domains of CD3 $\zeta$. Combined antitumor effects of the NKAB-ErbB2 molecule and the NKAR-NK-92 cells in vivo were investigated in a syngeneic tumor model in immunocompetent mice with ErbB2-positive murine glioblastoma cells, mimicking low or absent NKG2DL expression.

\section{MATERIALS AND METHODS}

\section{Cells and culture conditions}

Human MDA-MB453, MDA-MB468, and JIMT-1 breast carcinoma, LNT-229 glioblastoma, and HEK 293T embryonic kidney cells (all American Type Culture Collection [ATCC], Manassas, Virginia, USA), and murine B16-F10/ErbB2 melanoma ${ }^{26}$ and GL261/ErbB2 glioblastoma cells ${ }^{27}$ were cultured in Dulbecco's modifed Eagle's medium (DMEM; Lonza, Cologne, Germany), human K562 erythroleukemia cells (ATCC) in RPMI 1640 medium (Lonza). Supplements included 10\% heat-inactivated FBS, $2 \mathrm{mmol} / \mathrm{L}$ L-glutamine, $100 \mathrm{U} / \mathrm{mL}$ penicillin, $100 \mu \mathrm{g} / \mathrm{mL}$ streptomycin (Life Technologies, Darmstadt, Germany). Medium for GL261/ErbB2 cells additionally contained $0.4 \mathrm{mg} / \mathrm{mL}$ G418. Human NK-92 cells (kindly provided by NantKwest, Culver City, California, USA) were propagated in X-VIVO 10 medium (Lonza) supplemented with 5\% heat-inactivated human plasma (German Red Cross Blood Donation Service Baden-Württemberg-Hessen, Frankfurt, Germany) and $100 \mathrm{IU} / \mathrm{mL}$ interleukin (IL)-2 (Proleukin; Novartis Pharma, Nürnberg, Germany).

Peripheral blood NK (pNK) cells of healthy donors were isolated from buffy coats by Ficoll-Hypaque density gradient centrifugation using the RosetteSep human NK cell enrichment cocktail (STEMCELL Technologies, Cologne, Germany) according to the manufacturers' instructions. Purity of enriched cells was confirmed by flow cytometric analysis using BV421-conjugated antiCD56 and Phycoerythrin (PE)-conjugated anti-CD3 antibodies (BD Biosciences, Heidelberg, Germany), and ranged between $83 \%$ and $96 \%$. For ex vivo expansion, typically $1 \times 10^{6} \mathrm{NK}$ cells were cultured for up to 3 weeks in X-VIVO 10 medium supplemented with $5 \%$ heatinactivated human plasma, $500 \mathrm{IU} / \mathrm{mL}$ IL-2 and $50 \mathrm{ng} /$ mL IL-15 (PeproTech, Hamburg, Germany).

\section{Expression and purification of bispecific NKAB-ErbB2 antibodies}

The $\mathrm{IgG}_{4}$-based NKAB-ErbB2 sequence was designed by in silico assembly of an immunoglobulin heavy-chain signal peptide, an scFv of NKG2D-specific antibody KYK$2.0,{ }^{28}$ hinge, CH2 and $\mathrm{CH} 3$ domains of human $\mathrm{IgG}_{4}$ (UniProtKB-P01861, amino acid residues 104-327), a $\left(\mathrm{G}_{4} \mathrm{~S}\right)$, linker, and the ErbB2-specific scFv(FRP5) antibody fragment. ${ }^{29}{ }^{30}$ For the similar NKAB-ErbB2 ( $\left.\operatorname{IgG}_{1}\right)$ molecule, the hinge, $\mathrm{CH} 2$ and $\mathrm{CH} 3$ domains of human $\mathrm{IgG}_{1}$ were used (UniProtKB-P01857, amino acid residues 99-330). In an alternative protein design, the scFv antibody fragments within the $\mathrm{IgG}_{4}$-based NKAB-ErbB2 sequence were exchanged, resulting in NKAB-ErbB2 (rev) with reverse orientation of the binding domains. Codon-optimized fusion genes were de novo synthesized (GeneArt; Thermo Fisher Scientific, Darmstadt, Germany) and inserted into mammalian expression vector pcDNA3, resulting in plasmids pcDNA3-NKAB-ErbB2, pcDNA3-NKAB-ErbB2 $\left(\operatorname{IgG}_{1}\right)$, and pcDNA3-NKAB-ErbB2 (rev). As a control, monospecific mini-antibody FRP5-Fc was generated, which encompassed an immunoglobulin heavy-chain signal peptide, the ErbB2-specific scFv(FRP5) antibody fragment, and hinge, $\mathrm{CH} 2$ and $\mathrm{CH} 3$ domains of human $\operatorname{IgG}_{1}$. Recombinant antibodies were expressed in 
transiently transfected HEK $293 \mathrm{~T}$ cells and purified from culture supernatant by affinity chromatography using a HiTrap Protein-G column on an ÄKTA FPLC system (GE Healthcare Europe, Freiburg, Germany). Purity and integrity of NKAB antibodies were determined by sodium dodecyl sulphate-polyacrylamide gel electrophoresis (SDS-PAGE) and Coomassie staining, or immunoblotting with horseradish peroxidase (HRP)-conjugated anti-human IgG antibody (Sigma-Aldrich, Munich, Germany). Protein concentrations were determined using a Nanodrop 1000 spectrophotometer (Thermo Fisher Scientific).

\section{Expression of lineage markers, NKG2D and natural cytotoxicity receptors}

Expression of NKG2D and lineage markers by peripheral blood mononuclear cells (PBMCs) from healthy donors was assessed using PE-conjugated anti-NKG2D (Miltenyi Biotec, Bergisch Gladbach, Germany), BV421-conjugated anti-CD56, and allophycocyanin (APC)-conjugated antiCD3 (BD Biosciences) antibodies. Phenotypical characterization of ex vivo expanded primary NK cells was performed with BV421-conjugated anti-CD56, PE-conjugated anti-CD3, Alexa Fluor 647-conjugated anti-CD16, PE-conjugated anti-NKp30, Alexa Fluor 647-conjugated anti-NKp44 (all BD Biosciences), PE-conjugated antiNKG2D, and APC-conjugated anti-NKp46 (both Miltenyi Biotec) antibodies. In all staining procedures, a human Fc receptor blocking agent was included (BD Biosciences). Expression of NK-cell activating receptors by NK-92 and NKAR-NK-92 cells was determined using PE-conjugated anti-NKG2D, PE-conjugated anti-NKp30, APC-conjugated anti-NKp46 (all Miltenyi Biotec), and APC-conjugated anti-NKp44 (R\&D Systems, WiesbadenNordenstadt, Germany) antibodies. Flow cytometric analysis was performed with FACSCanto II or BD LSRFortessa flow cytometers (BD Biosciences), and data were analyzed using FACSDiva or FlowJo software V.10.0.7 (FlowJo, Ashland, Oregon, USA). Binding of NKAB-ErbB2 to lymphocytes was either investigated using purified NKABErbB2 followed by APC-conjugated secondary antibody (Dianova, Hamburg, Germany) or purified NKAB-ErbB2 covalently labeled with APC using a conjugation kit (Abcam, Cambridge, UK) according to the manufacturer's recommendations.

\section{Time-lapse imaging}

MDA-MB453 breast carcinoma cells as targets were transduced with a lentiviral vector encoding luciferase and superfolder green fluorescent protein (GFP) ${ }^{31}$ As effector cells, PBMCs were isolated from buffy coats as described previously and either cultured for 3 days in X-VIVO 10 medium supplemented with $5 \%$ heat-inactivated human plasma, $500 \mathrm{IU} / \mathrm{mL}$ IL-2 and $50 \mathrm{ng} / \mathrm{mL}$ IL-15, or on antiCD3 antibody-coated plates in RPMI 1640 medium supplemented with $10 \%$ heat-inactivated human plasma, $100 \mathrm{IU} /$ $\mathrm{mL}$ IL-2 and $1 \mu \mathrm{g} / \mathrm{mL}$ anti-CD28 antibody (BioLegend, Koblenz, Germany) to preferentially stimulate NK or T cells, respectively. GFP-positive target cells were seeded on poly-L-lysine-coated glass bottom dishes (MatTek, Ashland, Massachusetts, USA) and allowed to adhere for 2-8 hours. Effector cells were treated with human Fc block (BD Biosciences) and then stained with PE-conjugated anti-CD3 and BV421-conjugated anti-CD56 antibodies (BD Biosciences) (IL-2/IL-15-stimulated PBMCs), or PE-conjugated anti-CD3 and BV421-conjugated anti-CD8 (BioLegend) antibodies (anti-CD3/anti-CD28-stimulated PBMCs). Then stained PBMCs were added to the GFPpositive target cells in the presence of $0.64 \mathrm{nM}(100 \mathrm{ng} /$ $\mathrm{mL}$ ) APC-conjugated NKAB-ErbB2 protein. Time-lapse imaging was performed using a CQ1 Confocal Quantitative Image Cytometer (Yokogawa, Tokyo, Japan). Phasecontrast and fluorescent images were taken every $5 \mathrm{~min}$ at $\times 40$ magnification by sample excitation with lasers at $405,488,561$, and $640 \mathrm{~nm}$. Images were captured at four z-stacks and processed to maximum intensity projections (MIPs).

\section{Generation of NKAR-expressing effector cells}

The NKG2D-based chimeric antigen receptor NKAR consists of an immunoglobulin heavy-chain signal peptide, the NKG2D extracellular domain (UniProtKB-P26718, amino acid residues 82-216), a $\left(\mathrm{G}_{4} \mathrm{~S}\right)_{2}$ linker, a Myc tag and a modified CD8 $\alpha$ hinge region, ${ }^{30}$ followed by CD3 $\zeta$ transmembrane and intracellular domains. The codonoptimized NKAR sequence was de novo synthesized (GeneArt, Thermo Fisher Scientific) and inserted into lentiviral transfer plasmid pHR'SIN-cPPT-SIEW upstream of IRES and enhanced green fluorescent protein (EGFP) sequences, ${ }^{32}$ yielding vector pS-NKAR-IEW. VSV-G pseudotyped vector particles were produced and NK-92 cells were transduced as described ${ }^{30}$ NKAR-positive cells were enriched by sorting with a FACSAria fluorescenceactivated cell sorter (BD Biosciences), with selection based on EGFP expression and enhanced NKG2D signals detected with anti-NKG2D antibody (Clone 149810, R\&D Systems) followed by APC-coupled secondary antibody. NKAR expression by sorted cells was confirmed by SDSPAGE of cell lysates and immunoblotting with anti-CD3 $\zeta$ (6B10.2) or anti-CD8 $\alpha$ antibodies (H-160; both Santa Cruz Biotechnology, Heidelberg, Germany), followed by HRP-conjugated secondary antibody and chemiluminescent detection. Similarly, CAR-engineered primary $\mathrm{T}$ cells were derived by lentiviral transduction with the NKAR construct. Interaction of NKAR with soluble MICA (sMICA) was investigated by flow cytometry with recombinant His-tagged human MICA (Biozol, Eching, Germany) followed by APC-conjugated anti-His-tag antibody (BioLegend).

\section{Cytotoxicity assays}

Cytotoxicity of effector cells was analyzed in flow cytometry-based assays as described. ${ }^{30}$ Briefly, tumor cells were labeled with calcein violet AM (CV) (Invitrogen, Thermo Fisher Scientific) and incubated with effector cells at various effector to target $(\mathrm{E} / \mathrm{T})$ ratios for 3 hours 
at $37^{\circ} \mathrm{C}$ in the presence or absence of bispecific antibodies. Then $150 \mu \mathrm{L}$ of a $1 \mu \mathrm{g} / \mathrm{mL}$ propidium iodide (PI) solution was added to each sample before flow cytometric analysis in a FACSCanto II flow cytometer. Dead target cells were identified as CV and PI double positive. Spontaneous target cell lysis was subtracted to calculate specific cytotoxicity. Data were analyzed using FACSDiva software. Unless stated otherwise, experiments were conducted at least twice with technical triplicates in each case. For competition experiments, sMICA-Fc fusion protein (R\&D Systems) or recombinant human $\mathrm{IgG}_{4}$ protein (Biozol) was added to the cultures.

\section{In vivo tumor model}

Female C57BL/6N mice (Charles River, Sulzfeld, Germany) 6-8 weeks old were used for a syngeneic GL261/ErbB2 murine glioblastoma model. Mice were inoculated with $1 \times 10^{6}$ tumor cells at the right flank. Seven days later, animals with palpable tumors were randomized into groups of eight to nine animals and treated by peritumoral injection of $1 \times 10^{7}$ NKAR-NK-92 or parental NK-92 cells in $200 \mu \mathrm{L}$ of injection medium, with or without addition of $5 \mu \mathrm{g}$ of NKAB-ErbB2 antibody. Treatment was repeated two times per week for 3 weeks. Tumor growth was followed by caliper measurements at preset intervals, and tumor volumes were calculated using the formula: length $\times(\text { width })^{2} \times 0.5$. Symptom-free survival was assessed by daily inspection. Group sizes were chosen to allow statistical analysis of differences in symptom-free survival. All treated animals were included in the analysis. Animal experiments were approved by the responsible government committee (Regierungspräsidium Darmstadt, Darmstadt, Germany) and were conducted according to the applicable guidelines and regulations in the animal facility of Georg-Speyer-Haus.

To assess programmed cell death protein 1 (PD-1) expression by tumor-infiltrating lymphocytes, tumor-bearing mice from the different treatment groups were sacrificed. Tumor tissues were collected and single cell suspensions were prepared by incubating the minced tissues with collagenase (Sigma-Aldrich) at $37^{\circ} \mathrm{C}$ for 1 hour. Likewise, peripheral blood was collected and single cell suspensions of splenocytes were prepared. Erythrocytes in blood and splenocyte samples were lysed using BD Pharm Lyse buffer (BD Biosciences). The prepared cells were stained with PE-conjugated anti-mouse PD-1 (BioLegend), APCconjugated anti-mouse $\mathrm{CD} 8 \alpha$ (eBioscience, Thermo Fisher Scientific), Pacific Blue-conjugated anti-mouse CD3 $\varepsilon$ and PerCP-Cy5.5-conjugated anti-mouse CD4 (both $\mathrm{BD}$ Biosciences) antibodies in the presence of mouse Fc block (BD Biosciences). Dead cells were excluded by staining with eFluor780 (eBioscience, Thermo Fisher Scientific). A PE-conjugated isotype control antibody (BD Biosciences) was used to differentiate non-specific background signals. Flow cytometric analysis was performed with a BD LSRFortessa flow cytometer and data were analyzed using FlowJo software.

\section{Statistical analysis}

Data were analyzed by two-tailed unpaired or paired Student's t-test and two-way analysis of variance. Symptomfree survival was analyzed by Kaplan-Meier plot and logrank (Mantel-Cox) test. P values of $<0.05$ were considered statistically significant. Prism V.9 software (GraphPad Software, La Jolla, California, USA) was used for all statistical calculations.

\section{RESULTS}

\section{Generation of a bispecific antibody binding to NKGD2 and} ErbB2

To target NKG2D-expressing lymphocytes to the tumorassociated antigen ErbB2, we designed a bispecific antibody similar in structure and molecular mass to an IgG molecule. This fusion protein (termed NKAB-ErbB2) carries an N-terminal $\mathrm{scFv}$ antibody domain derived from an NKG2D-specific antibody, ${ }^{28}$ and a second C-terminal scFv domain derived from ErbB2-specific antibody FRP5. ${ }^{29}$ To enable dimerization and provide flexibility between the two scFv domains, they were linked via the hinge, CH2 and $\mathrm{CH} 3$ domains of human $\mathrm{IgG}_{4}$ and a $\left(\mathrm{G}_{4} \mathrm{~S}\right)_{2}$ peptide sequence (figure $\left.1 \mathrm{~A}\right)$. For expression as a secreted protein, the codon-optimized antibody sequence was fused to an immunoglobulin heavy-chain signal peptide sequence in a pcDNA3 expression plasmid. The recombinant NKAB-ErbB2 molecule was then expressed in transiently transfected HEK 293T cells and purified from culture supernatant by protein $\mathrm{G}$ affinity chromatography. SDS-PAGE and immunoblot analysis under reducing and non-reducing conditions confirmed purity and identity of the protein and revealed expression of the molecule as a tetravalent disulfide-linked homodimer, with only a minor fraction present in monomeric form under non-reducing conditions (figure 1B). Flow cytometric analysis demonstrated specific binding of the NKAB antibody to ErbB2-expressing but NKG2D-negative MDA-MB453 breast carcinoma and ErbB2-negative but NKG2D-positive NK-92 cells, but not to MDA-MB468 breast carcinoma cells which lack expression of ErbB2 and NKG2D (figure 1C and online supplemental figure S1). This demonstrates that the NKG2D-specific and ErbB2-specific binding domains in the molecule were both functionally active.

\section{Effect of bispecific NKAB-ErbB2 antibody on the lytic activity of NKG2D-expressing peripheral blood lymphocytes and NK cells}

Next, we investigated whether NKAB-ErbB2 influences the antitumor activity of NKG2D-positive peripheral blood lymphocytes in in vitro cytotoxicity assays using freshly isolated PBMCs from three healthy donors. Proportions of effector lymphocytes varied, depending on the individual donor, with $\mathrm{NK}$ cells $\left(\mathrm{CD} 56^{+} \mathrm{CD}^{-}\right)$ranging from $3.5 \%$ to $6.1 \%$, CD56-positive T cells $\left(\mathrm{CD} 56^{+} \mathrm{CD}^{+}\right)$ ranging from $3.6 \%$ to $13.9 \%$, and CD56-negative $\mathrm{T}$ cells $\left(\mathrm{CD}^{-} 6^{-} \mathrm{CD}^{+}\right)$ranging from $55.4 \%$ to $63.2 \%$ (figure $2 \mathrm{~A}$ ). 
A

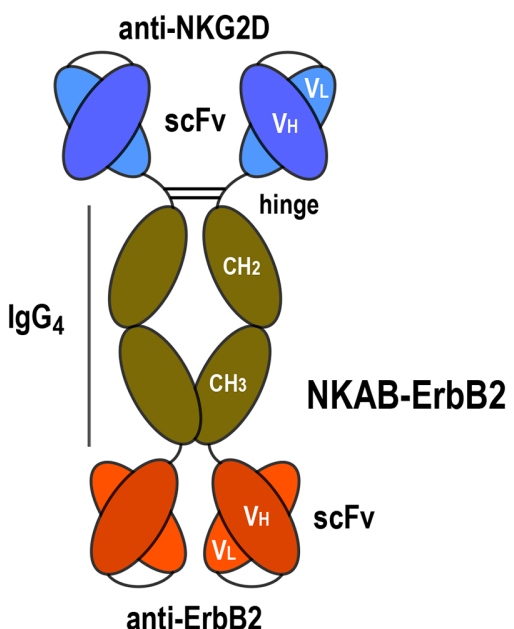

B NKAB-ErbB2

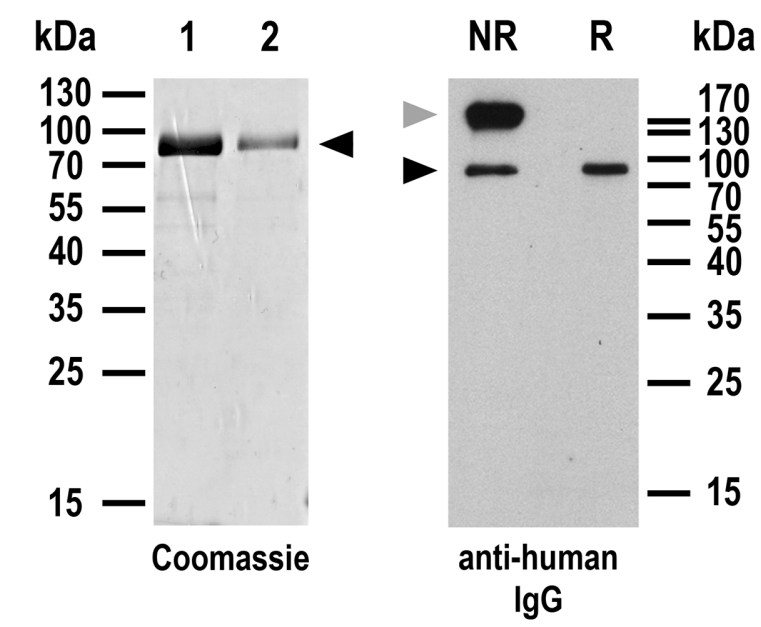

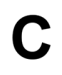

MDA-MB453 $\mathrm{ErbB2}^{+}$/NKG2D- $^{-}$

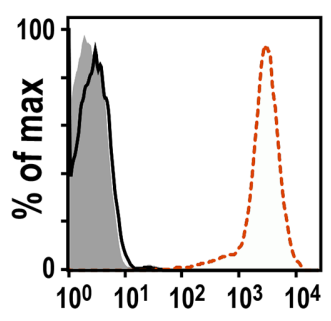

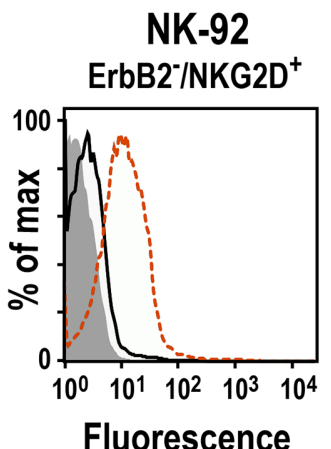

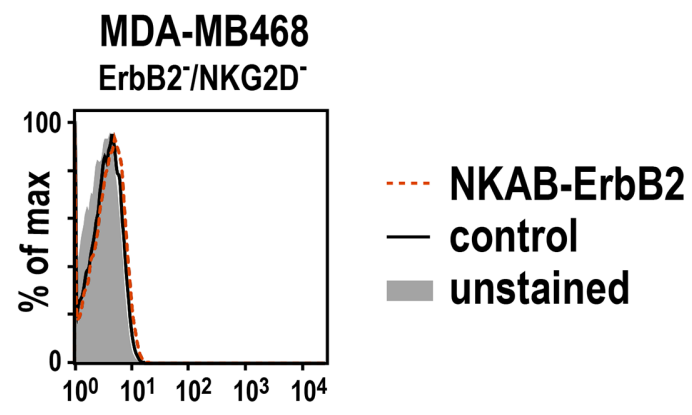

Fluorescence

Figure 1 Expression and purification of bispecific NKAB-ErbB2 antibody. (A) Schematic representation of bispecific antibody NKAB-ErbB2 consisting of an N-terminal NKG2D-specific scFv antibody fragment, hinge, $\mathrm{CH} 2$ and $\mathrm{CH} 3$ domains of human $\operatorname{lgG}_{4}, a\left(G_{4}\right)_{2}$ linker, and a C-terminal ErbB2-specific scFv antibody fragment. Disulfide bridges connecting the monomers within the homodimeric molecule are indicated by lines. (B) Analysis of elution fractions 1 and 2 after purification of NKAB-ErbB2 antibody via protein-G affinity chromatography from culture supernatants of transiently transfected HEK 293T cells by SDSPAGE under reducing conditions and Coomassie staining (left), and immunoblot analysis of purified NKAB-ErbB2 protein after SDS-PAGE under NR or reducing conditions (R) with HRP-conjugated anti-human IgG antibody followed by chemiluminescent detection (right). The positions of NKAB-ErbB2 monomers and homodimers are indicated by black and gray arrowheads, respectively. (C) Binding of purified NKAB-ErbB2 to ErbB2 and NKG2D was investigated by flow cytometry with ErbB2-positive but NKG2D-negative MDA-MB453 breast carcinoma cells, ErbB2-negative but NKG2D-positive NK-92 cells, and doublenegative MDA-MB468 breast carcinoma cells (dashed red lines). Unstained cells (filled areas) and cells only incubated with secondary antibody (solid black lines) were included as controls. NKG2D, natural killer group 2D; NR, non-reducing; scFv, single-chain fragment variable.

All lymphocyte subpopulations tested showed NKG2D surface expression (figure 2B). Thereby, purified NKABErbB2 displayed binding to NKG2D-positive NK cells, and CD56-positive and CD56-negative T cells in a manner very similar to that of a commercial anti-NKG2D antibody (online supplemental figure S2). In the absence of bispecific antibody, unstimulated PBMCs displayed little to moderate cytotoxicity against MDA-MB453 breast carcinoma cells ranging from 3.4 (D1) to $15.3 \%$ (D3) specific cell killing after 3 hours of coincubation at an $\mathrm{E} / \mathrm{T}$ ratio of 10:1 (figure 2C), likely due to NKG2D-mediated activation of the effector cells by NKG2DLs endogenously expressed by the target cells (online supplemental figure S1). In the presence of NKAB-ErbB2 antibody, cytotoxicity against the ErbB2-overexpressing cancer cells increased in a dose-dependent manner, with maximum cell killing of 2.0-fold to 2.6-fold over baseline reached at an antibody concentration of $0.64 \mathrm{nM}(100 \mathrm{ng} / \mathrm{mL}$; D1 and D2) or $3.2 \mathrm{nM}(500 \mathrm{ng} / \mathrm{mL}$, D3) (figure 2D). Cytotoxic activity decreased again at NKAB-ErbB2 concentrations above saturation of bispecific binding, indicative of competition by free antibody molecules. To investigate whether different NKG2D-positive cell types can contribute to target cell killing, the interaction of PBMCs prestimulated to preferentially activate NK or T cells with MDAMB453 breast cancer cells was investigated in time-lapse imaging experiments. Thereby both, NK cells and CD8 ${ }^{+}$ $T$ cells induced lysis of tumor cells opsonized by NKABErbB2 as indicated by membrane blebbing and formation of apoptotic bodies, with some of the NK cells exhibiting serial target cell killing (figure 3). 
A

D1

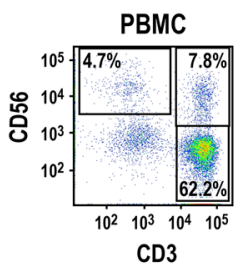

D2

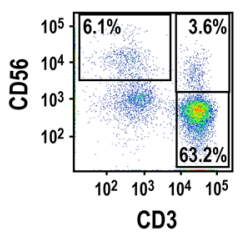

D3

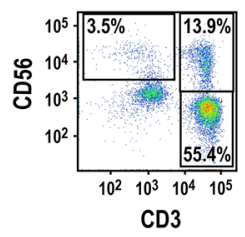

B
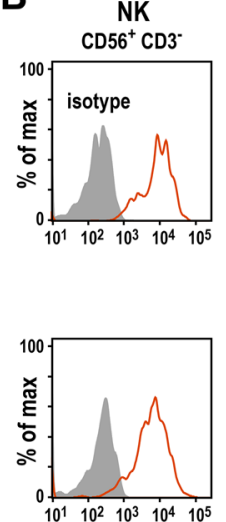

$\begin{array}{lllll}10^{1} & 10^{2} & 10^{3} & 10^{4} & 10^{5}\end{array}$

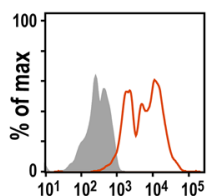

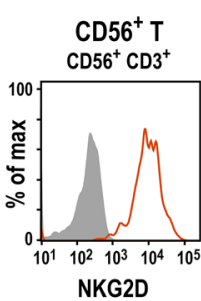
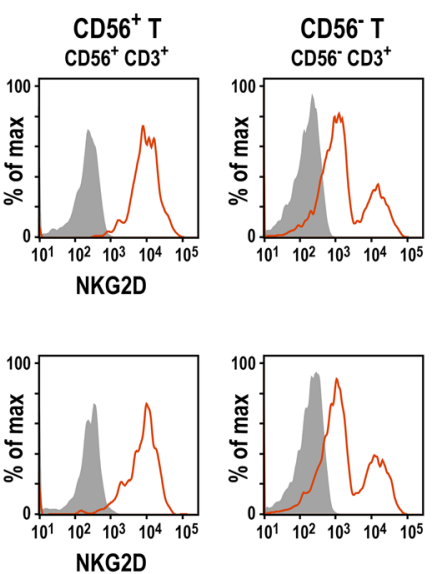

NKG2D

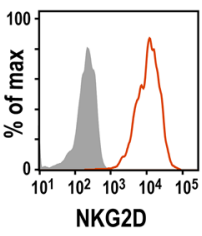

C
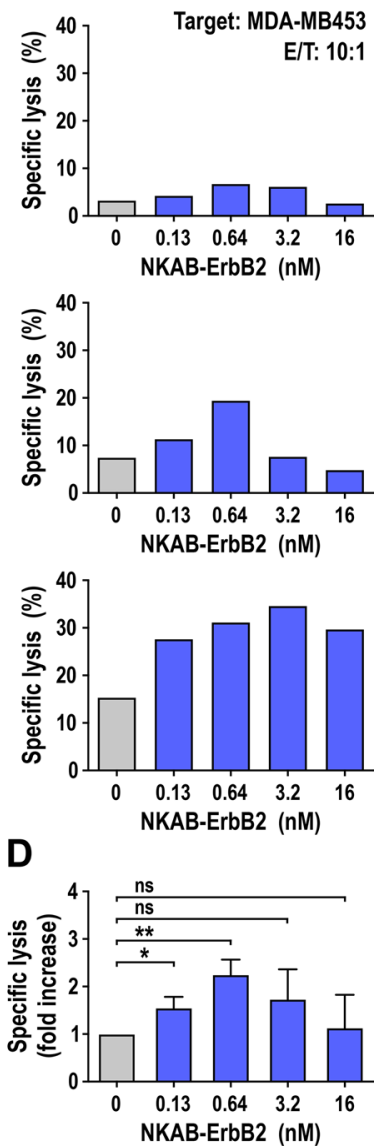

Figure 2 NKAB-ErbB2-mediated redirection of donor-derived lymphocytes to ErbB2-expressing cancer cells. (A) Proportions of NK (CD56 $\left.{ }^{+} \mathrm{CD}^{-}\right)$, CD56-positive $\left(\mathrm{CD}^{-} 6^{+} \mathrm{CD}^{+}\right)$and $\mathrm{CD} 56$-negative T cells $\left(\mathrm{CD}^{-} 6^{-} \mathrm{CD}^{+}\right)$in freshly isolated PBMCs from healthy donors (D1-D3; B,C). (B) Expression of NKG2D by NK, CD56-positive and CD56-negative T-cell populations of the cells (A) gated according to their CD56 and CD3 expression was analyzed by flow cytometry with anti-NKG2D antibody (solid red lines). An irrelevant antibody of the same isotype served as control (filled areas). (C) Cytotoxicity of the PBMCs (A) against ErbB2-expressing MDA-MB453 breast carcinoma cells in the absence of bispecific antibody (gray bars) or in the presence of increasing concentrations of recombinant NKAB-ErbB2 (blue bars) was investigated in flow cytometry-based cytotoxicity assays after coincubation at an E/T of 10:1 for 3 hours. (D) Normalized cytotoxicity data for PBMCs in the presence of NKABErbB2 shown in (C) are plotted as fold increase in specific lysis in comparison to PBMCs alone. Mean values \pm SD are shown; $\mathrm{n}=3$ individual donors. Data were analyzed by two-tailed unpaired Student's $t$-test. ${ }^{*} \mathrm{P}<0.05,{ }^{*} \mathrm{P}<0.01$. ns: $\mathrm{p}>0.05$. $E / T$, effector to target ratio; NK, natural killer; NKG2D, natural killer group 2D; ns, not significant; PBMC, peripheral blood mononuclear cell.

In a next set of experiments, we evaluated the activity of the bispecific NKAB-ErbB2 molecule with purified and ex vivo expanded pNK cells. To allow direct comparison between NKG2D- and CD16-mediated cytotoxicity, we included an FRP5-Fc mini-antibody consisting of the same ErbB2-specific scFv domain used for NKAB-ErbB2 but linked to the CD16-binding Fc portion of human $\operatorname{IgG}_{1}$ (online supplemental figure S3). pNK cells from three different donors were expanded for 2-3 weeks in medium containing IL-2 and IL-15, with around $75 \%-85 \%$ of cells in the resulting populations coexpressing NKG2D and CD16 (figure 4A). On coincubation with MDA-MB453 tumor cells for 3 hours at an E/T ratio of 5:1, pNK cells from all donors demonstrated moderate baseline cytotoxicity in the absence of antibody, which depending on the donor was enhanced in a concentration-dependent manner to up to 3.9-fold by NKAB-ErbB2, with maximum cell killing again reached at $0.64 \mathrm{nM}(100 \mathrm{ng} / \mathrm{mL}$; D4 and D6) or $3.2 \mathrm{nM}(500 \mathrm{ng} / \mathrm{mL}$, D5) (figure 4B). Also antibody-dependent cell-mediated cytotoxicity triggered by FRP5-Fc through activation of CD16 resulted in increased antitumor activity of pNK cells, although for all donors to a lower degree than NKAB-ErbB2 (figure 4C). To clearly distinguish NKG2D- and CD16-mediated effects, we based the NKAB-ErbB2 molecule on $\operatorname{IgG}_{4}$, which does not bind CD16 with high affinity. To assess potential additive effects by ligating both NKG2D and CD16 to ErbB2-positive tumor cells, we also generated an NKAB-ErbB2 $\left(\operatorname{IgG}_{1}\right)$ molecule (online supplemental figure S3) and compared its activity to that of the original $\mathrm{IgG}_{4}$-based NKAB-ErbB2 antibody with pNK cells from another three donors. Thereby a very similar increase in cytotoxic activity of pNK cells against ErbB2-positive breast cancer cells was found for NKAB-ErbB2 and NKABErbB2 $\left(\operatorname{IgG}_{1}\right)$. 
A
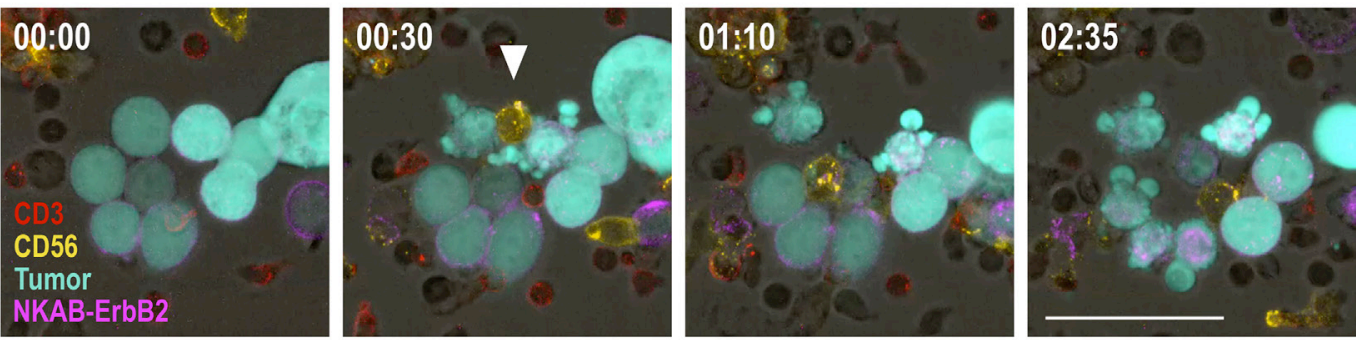

B
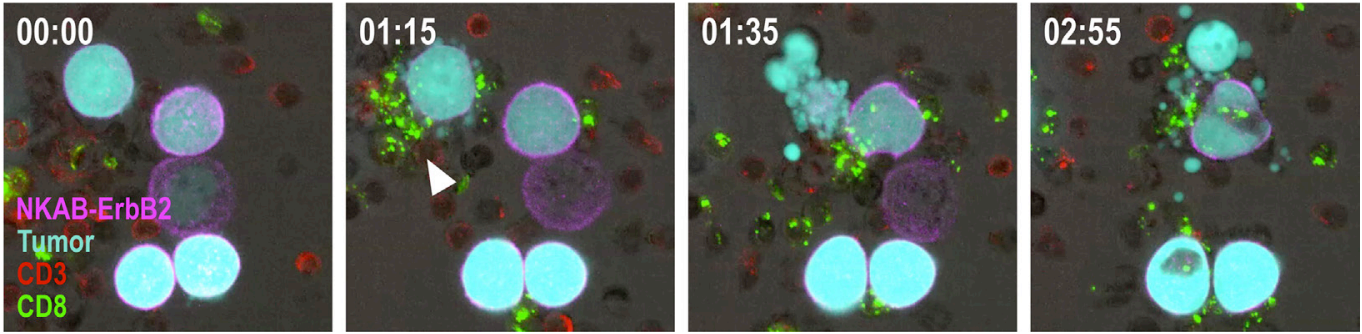

Figure 3 Interaction of peripheral blood lymphocytes with breast cancer cells in the presence of bispecific NKAB-ErbB2 antibody. PBMCs from a healthy donor were prestimulated with IL-2 and IL-15 (A), or IL-2 and anti-CD3 and anti-CD28 antibodies (B), to preferentially stimulate NK or T cells, respectively. Prestimulated PBMCs were either stained with anti-CD3 (red) and anti-CD56 (yellow) (A), or anti-CD3 (red) and anti-CD8 (green) antibodies (B), before incubation with GFP-expressing MDA-MB453 breast carcinoma cells (turquoise) in the presence of $0.64 \mathrm{nM}(100 \mathrm{ng} / \mathrm{mL})$ of NKAB-ErbB2 protein (purple). Phase-contrast and fluorescent images were taken every 5 min at $\times 40$ magnification with a CQ1 Confocal Quantitative Image Cytometer. Representative images of individual fields taken at the indicated time points (hours: min) are shown. An NK cell and a CD8 ${ }^{+} \mathrm{T}$ cell inducing lysis of target cells opsonized by NKAB-ErbB2 are indicated by white arrowheads (A,B), respectively, with the NK cell exhibiting serial target cell killing. Scale bar: $50 \mu \mathrm{m}$. GFP, green fluorescent protein; IL, interleukin; NK, natural killer; PBMC, peripheral blood mononuclear cell.

\section{Enhancement of NK-cell activity by an NKG2D-based CAR}

CARs which employ NKG2D for recognition of stressinduced NKG2DLs can enhance cytotoxic activity of CARengineered $\mathrm{T}$ and NK cells towards tumor cells of various origins. ${ }^{10}{ }^{11}$ Since NKG2D is a type II transmembrane glycoprotein with an extracellular C-terminus, in NKG2Dbased CARs heterologous signaling domains derived from type I transmembrane proteins such as CD3 $\zeta$ are either added in a reverse orientation with regard to the plasma membrane in front of the cytoplasmic N-terminus of NKG2D, or an N-terminally truncated NKG2D ectodomain is artificially extended at the C-terminus with heterologous hinge and transmembrane regions followed by the respective cytoplasmic signaling motif, with both types of CARs being functional. ${ }^{11}$ Here we followed the latter type I transmembrane protein design and generated a CAR molecule (termed NKAR) that harbors an immunoglobulin heavy-chain signal peptide and the extracellular domain of human NKG2D at the N-terminus, fused to transmembrane and intracellular domains of CD3 $\zeta$ via a flexible linker, a Myc tag and an optimized CD8 $\alpha$ hinge region (figure 5A) ${ }^{30}$ For coexpression of the CAR sequence together with EGFP as a marker, VSV-G pseudotyped lentiviral vector particles were generated and used for transduction of human NK-92 cells as a clinically relevant model. ${ }^{33}$ Resulting NKAR-NK-92 cells were enriched by flow cytometric cell sorting. NKAR expression was examined by SDS-PAGE under non-reducing conditions and immunoblot analysis with $\mathrm{CD} 3 \zeta$ - and $\mathrm{CD} 8 \alpha$-specific antibodies, revealing the presence of NKAR monomers, disulfide-linked NKAR-NKAR homodimers and NKARCD3 $\zeta$ heterodimers (figure 5B). Surface expression of the NKAR molecule was confirmed by flow cytometry, identified by a markedly increased NKG2D signal in NKAR-NK-92 cells when compared with parental NK-92 (figure 5C). NKAR expression also led to increased levels of NKp30, while NKp44 and NKp46 were not or only marginally affected. This may be due to a stabilizing effect

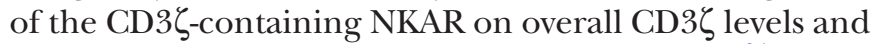
NKp30, which associates with CD3 $\zeta$ for signaling. ${ }^{34}$ NKAR expression resulted in strongly enhanced cytotoxicity of NKAR-NK-92 cells against K562 leukemia cells which express different NKG2DLs (figure 5D and online supplemental figure S1), indicating that the CAR molecule was functional.

\section{Synergistic effects of NKAB-ErbB2 and NKAR}

Next, we investigated the activity of NKAR-NK-92 cells combined with NKAB-ErbB2 against MDA-MB453 breast cancer cells, which express high levels of ErbB2 and different NKG2DLs (online supplemental figure S1) but are largely resistant to parental NK-92 cells. ${ }^{30}$ As seen with K562 target cells, NKAR-NK-92 cells already displayed increased lysis of MDA-MB453 cells in the absence of NKAB-ErbB2 (11.2\% vs $2.1 \%$ of specific killing at an E/T ratio of 5:1), which was markedly enhanced to more than 
A

D4

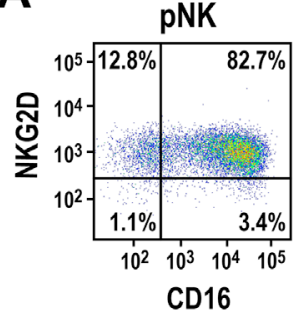

55

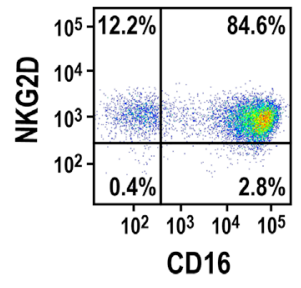

D6

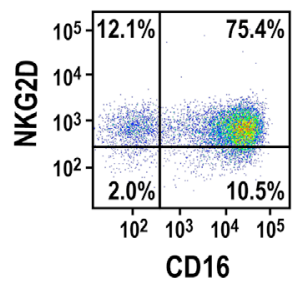

B
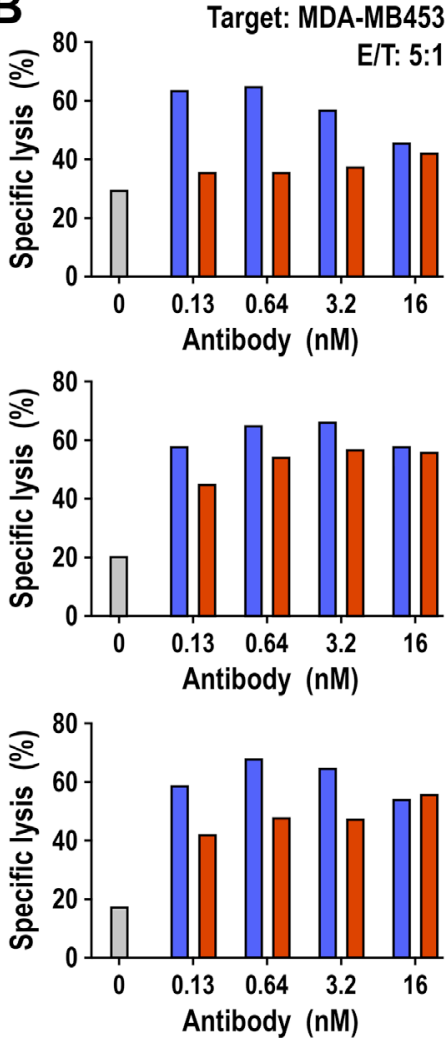

NKAB-ErbB2

FRP5-Fc

control

\section{C}
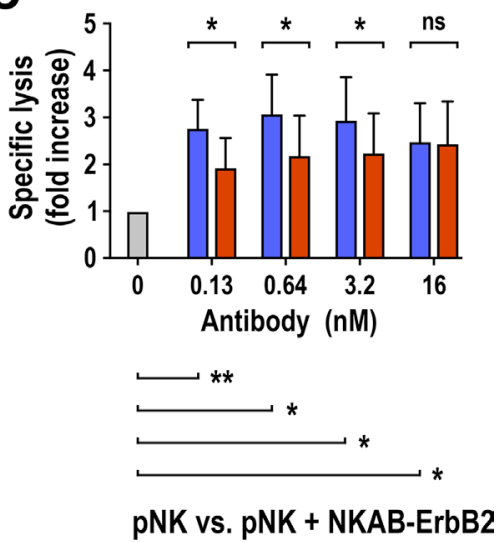

Figure 4 Effect of NKAB-ErbB2 on the cell killing activity of donor-derived NK cells. (A) Expression of NKG2D and CD16 by the ex vivo expanded pNK cells from healthy donors (D4-D6, B) was analyzed by flow cytometry with anti-NKG2D and anti-CD16 antibodies as indicated. (B) Cytotoxicity of the pNK cells (A) against ErbB2-expressing MDA-MB453 breast carcinoma cells in the absence of bispecific antibody (gray bars) or in the presence of increasing concentrations of recombinant NKAB-ErbB2 (blue bars) or ErbB2-specific FRP5-Fc IgG1 mini-antibody (red bars) was investigated in flow cytometry-based cytotoxicity assays after coincubation at an E/T of 5:1 for 3 hours. (C) Normalized cytotoxicity data for pNK cells in the presence of NKABErbB2 or FRP5-Fc (B) are plotted as fold increase in specific lysis in comparison to pNK alone. Mean values $\pm S D$ are shown; $n=3$ individual donors. Data were analyzed by two-tailed unpaired Student's t-test. ${ }^{*} P<0.05,{ }^{* *} P<0.01$. ns: $p>0.05$. E/T, effector to target ratio; NK, natural killer; NKG2D, natural killer group 2D; ns, not significant; pNK, peripheral blood NK.

$60 \%$ specific lysis in the presence of $0.16-0.64 \mathrm{nM}$ (25 to $100 \mathrm{ng} / \mathrm{mL}$ ) of NKAB-ErbB2 (figure $6 \mathrm{~A}$ ). As observed with donor-derived PBMCs, cytotoxic activity of NKARNK-92 cells decreased again gradually at NKAB-ErbB2 concentrations above $0.64 \mathrm{nM}$, likely due to competition of productive cross-linking of effector and target cells by free antibody molecules. Addition of NKAB-ErbB2 also increased cytotoxicity of parental NK-92 cells against MDA-MB453 cells in this short-term assay, although not to meaningful levels. This was likely due to the limited amount of endogenous NKG2D expressed by NK-92 (see figure 5C). Demonstrating a large degree of flexibility of the NKAB protein design, the same synergy in the killing of MDA-MB453 cells seen with NKAR-NK-92 and the original NKAB-ErbB2 was observed when the CAR-NK cells were combined with recombinant NKAB-ErbB2 (rev), a molecule in which the positions of NKG2D- and ErbB2specific binding domains relative to each other were exchanged (online supplemental figure S4).

To investigate whether the combined effect of NKAB-ErbB2 and NKAR-expressing effector cells can consistently be achieved with ErbB2-positive cells of solid tumor origin, we tested specific cytotoxicity of
NKAR-NK-92 cells at increasing E/T ratios with MDAMB453 and JIMT-1 breast cancer cells, and LNT-229 glioblastoma cells in the absence or presence of $0.16 \mathrm{nM}$ $(25 \mathrm{ng} / \mathrm{mL})$ NKAB-ErbB2. For comparison, MDAMB468 breast cancer cells were included, which also harbor NKG2DLs but are negative for ErbB2 (online supplemental figure $\mathrm{S} 1$ ). Even at the highest $\mathrm{E} / \mathrm{T}$ ratio applied, the four tested cell lines proved largely resistant to parental NK-92 cells, which was not changed significantly by the addition of NKAB-ErbB2 (figure 6B). In contrast, NKAR-NK-92 cells killed the NKG2DL-positive targets with high efficiency, which in the case of the ErbB2-expressing tumor cells was further enhanced in a synergistic manner by NKAB-ErbB2. Importantly, while also displaying markedly enhanced sensitivity to NKARNK-92 cells, the addition of NKAB-ErbB2 did not further increase cytotoxicity of NKAR-NK-92 against ErbB2negative MDA-MB468 cells, underscoring the specificity of the NKAB-ErbB2 effect (figure 6B). In the case of ErbB2-expressing targets, NKAB-ErbB2-mediated activation of the NKG2D-CAR did not only trigger selective cytotoxicity but also induced marked upregulation of proinflammatory cytokines such as IFN- $\gamma$, which is 
A

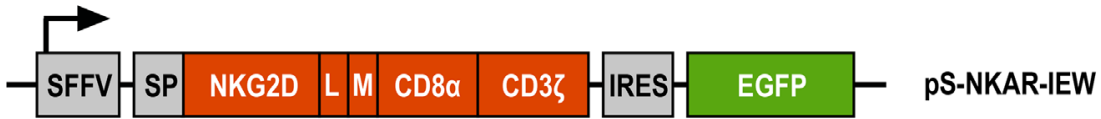

B

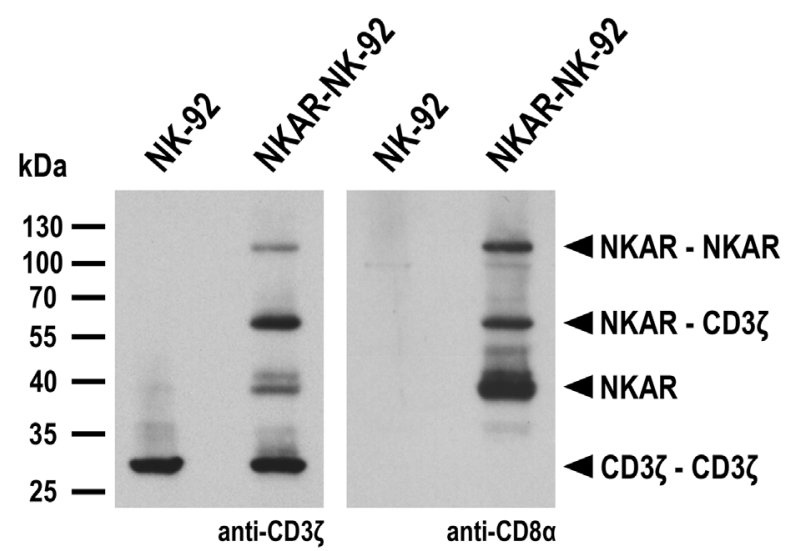

D

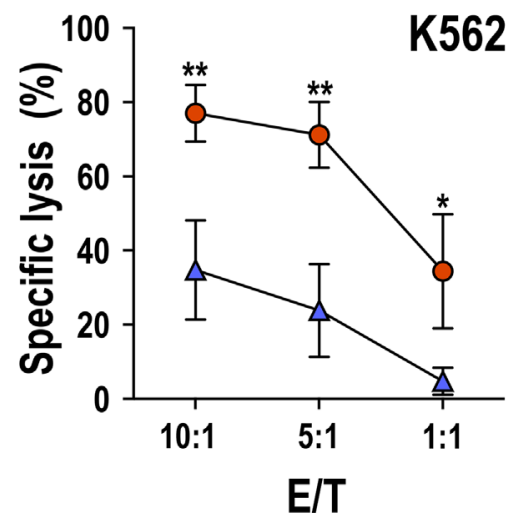

C
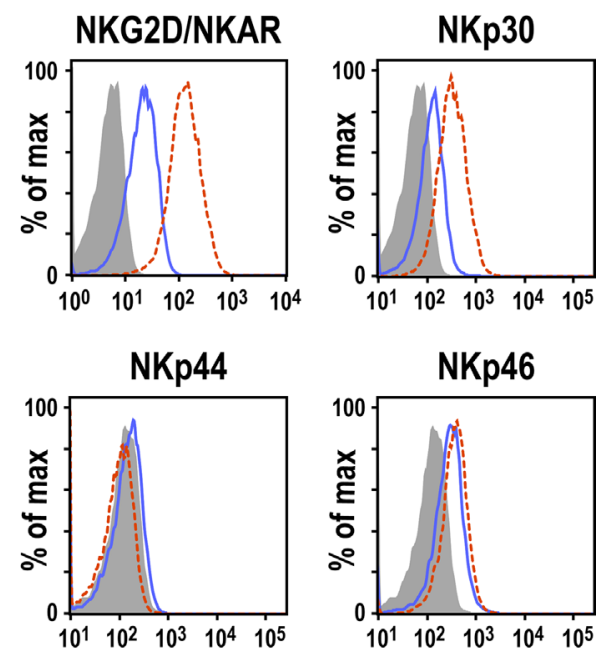

Fluorescence

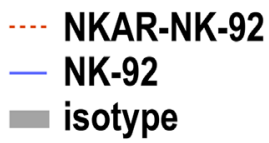

Figure 5 Generation of NKAR-NK-92 cells. (A) Lentiviral transfer plasmid encoding the NKG2D-based chimeric antigen receptor NKAR under the control of the SFFV promoter. The receptor consists of an immunoglobulin heavy-chain SP, the extracellular domain of NKG2D (amino acid residues 82-216), a flexible $\left(G_{4} s\right)_{2} L$, an $M$ tag, a CD8 $\alpha$ hinge region (CD8 $\alpha$ ),

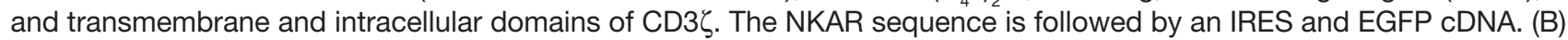
Expression of NKAR by sorted NKAR-NK-92 cells was analyzed by SDS-PAGE of whole cell lysate under non-reducing conditions and immunoblotting with $\mathrm{CD} 3 \zeta$-specific (left) and CD8 $\alpha$-specific antibodies (right), followed by HRP-conjugated secondary antibodies and chemiluminescent detection. Lysate of parental NK-92 cells was included as control. The positions of NKAR homodimers and monomers, CD3 $\zeta$ homodimers, and NKAR-CD3 $\zeta$ heterodimers are indicated by arrowheads. (C) Expression of the activating NK-cell receptors NKG2D and NKAR, NKp30, NKp44, and NKp46 in sorted NKAR-NK-92 (dashed red lines) and unmodified parental NK-92 cells (solid blue lines) was analyzed by flow cytometry using receptor-specific antibodies. NK-92 cells stained with irrelevant antibodies of the same isotype served as controls (filled areas). (D) Cytotoxicity of NKAR-NK-92 (red circles) and parental NK-92 cells (blue triangles) against K562 erythroleukemia cells was investigated in flow cytometry-based cytotoxicity assays after coincubation at different $E / T s$ for 3 hours. Mean values $\pm S D$ are shown; $n=3$ technical replicates from a representative experiment. Data were analyzed by two-tailed unpaired Student's t-test. ${ }^{*} \mathrm{P}<0.05$, ${ }^{* *} \mathrm{P}<0.01$. EGFP, enhanced green fluorescent protein; E/T, effector to target ratio; IRES, internal ribosome entry site; L, linker; M, Myc; NK, natural killer; NKG2D, natural killer group 2D; SFFV, spleen focus-forming virus; SP, signal peptide.

a hallmark of NK-cell activation (online supplemental figure S5).

Initially, the concept of targeting tumor cells through an NKG2D-based CAR was developed using genetically engineered $\mathrm{T}$ cells. ${ }^{9}$ To investigate whether the cytotoxicity of such CAR-T cells can also be enhanced by the NKAB-ErbB2 molecule, donor-derived $\mathrm{T}$ cells were transduced with the NKAR construct, and cytotoxicity of the resulting cell population against ErbB2-positive MDA-MB453 cells was tested in the absence or presence of $0.64 \mathrm{nM}(100 \mathrm{ng} / \mathrm{mL})$ NKAB-ErbB2. Thereby, similar to our findings with NK-92-derived NKAR-NK cells, the expression of NKAR on its own already increased cytotoxicity of NKAR-T cells against NKG2DL-positive targets, 
A

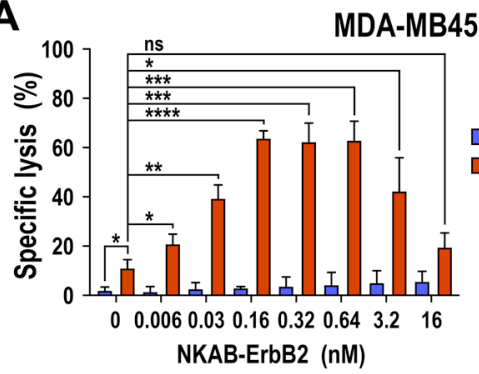

B

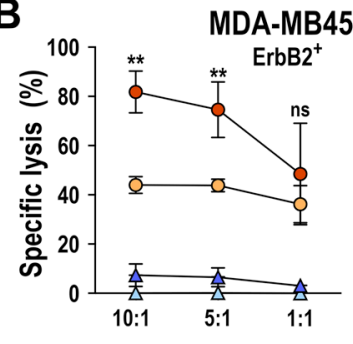

C

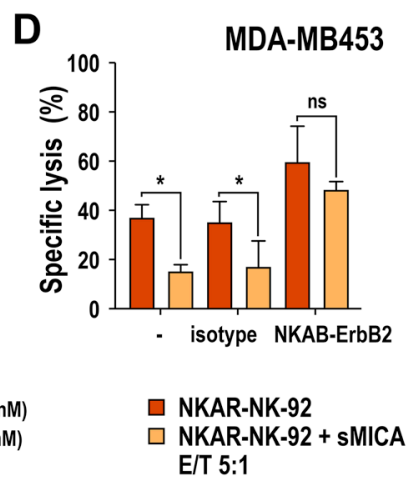

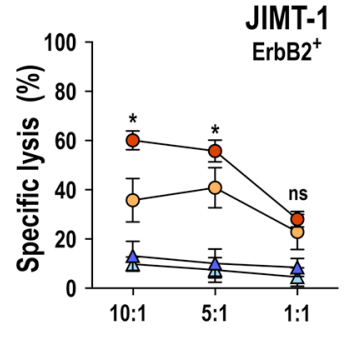

$\triangle$ NK-92

$\triangle$ NK-92 + NKAB-ErbB2
MDA-MB468

ErbB2"
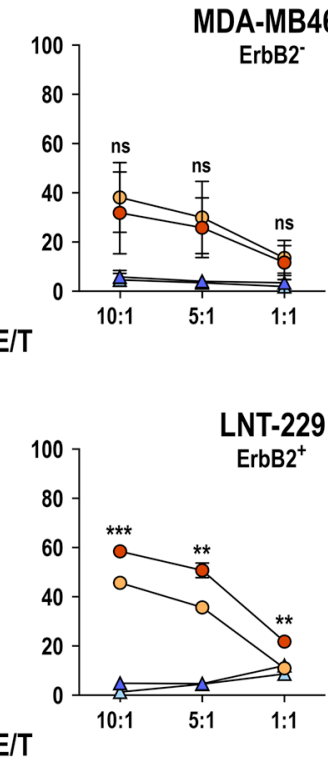

-o- NKAR-NK-92

- NKAR-NK-92 + NKAB-ErbB2

Figure 6 Enhancement of NKAR-NK-92 cytotoxicity by NKAB-ErbB2. (A) The effect of NKAB-ErbB2 on specific cytotoxicity of NKAR-NK-92 cells against ErbB2-positive MDA-MB453 breast carcinoma cells was determined in flow cytometry-based cytotoxicity assays after coincubation at an E/T of 5:1 for 3 hours in the absence or presence of increasing NKAB-ErbB2 concentrations (red bars). Parental NK-92 cells were included for comparison (blue bars). Mean values $\pm S D$ are shown; $n=3$ technical replicates from a representative experiment. Data were analyzed by two-tailed unpaired Student's t-test (shown for NKAR-NK-92 vs NK-92 in the absence of NKAB-ErbB2 and NKAR-NK-92 in the absence vs the presence of increasing concentrations of NKAB-ErbB2). ${ }^{\star} P<0.05,{ }^{\star \star} \mathrm{P}<0.01,{ }^{\star \star \star} \mathrm{P}<0.001$, ${ }^{\star \star \star \star} \mathrm{P}<0.0001$. ns: $\mathrm{p}>0.05$. (B) Cytotoxicity of NKAR-NK-92 (orange circles) and NK-92 cells (light blue triangles) in the absence, and NKAR-NK-92 (red circles) and NK-92 cells (dark blue triangles) in the presence of $0.16 \mathrm{nM}(25 \mathrm{ng} / \mathrm{mL})$ of NKAB-ErbB2 against MDA-MB453, MDA-MB468 and JIMT-1 breast carcinoma cells and LNT-229 glioblastoma cells was investigated in flow cytometry-based cytotoxicity assays after coincubation at different $E / T$ ratios for 3 hours. Mean values $\pm S D$ are shown; $n=3$ technical replicates from a representative experiment. Data were analyzed by two-tailed unpaired Student's t-test (shown for NKAR-NK-92+NKAB-ErbB2 vs NKARNK-92). ${ }^{*} P<0.05,{ }^{* \star} P<0.01,{ }^{* * *} P<0.001$. ns: $p>0.05$. (C) The ability of NKAB-ErbB2 to compete binding of sMICA to NKARNK-92 cells was determined by flow cytometry with APC-conjugated anti-His-tag antibody after incubation of cells with $2.5 \mu \mathrm{g} /$ $\mathrm{mL}$ of His-tagged sMICA in the absence (solid black line) or presence of $1.6 \mathrm{nM}(0.25 \mu \mathrm{g} / \mathrm{mL})$ or $16 \mathrm{nM}(2.5 \mu \mathrm{g} / \mathrm{mL})$ of NKABErbB2 (dashed red lines) as indicated. Cells treated only with secondary antibody served as control (filled area). (D) Inhibition of NKAR-NK-92 cell killing activity by $2.5 \mu \mathrm{g} / \mathrm{mL}$ of sMICA-Fc protein and restoration by addition of $0.16 \mathrm{nM}(25 \mathrm{ng} / \mathrm{mL})$ of NKABErbB2 were investigated in flow cytometry-based cytotoxicity assays after coincubation with MDA-MB453 target cells at an E/T ratio of $5: 1$ for 3 hours. Recombinant human $\operatorname{lgG}_{4}$ protein $(25 \mathrm{ng} / \mathrm{mL})$ served as isotype control. Mean values $\pm S D$ are shown; $n=3$ technical replicates from a representative experiment. Data were analyzed by two-tailed paired Student's t-test. ${ }^{*} \mathrm{P}<0.05$. ns: p $>0.05$. E/T, effector to target ratio; ns, not significant; sMICA, soluble MICA.

while enhanced cytotoxicity against ErbB2-positive tumor cells lacking human NKG2DLs was dependent on the simultaneous presence of NKAB-ErbB2 (online supplemental figure S6).

\section{Restoration of SMICA-inhibited NKAR functionality by NKAB- ErbB2}

Proteolytic shedding of NKG2DLs like MICA has been identified as a mechanism for cancer cells to evade NKG2Dmediated immune surveillance. ${ }^{11}{ }^{23}$ To test whether this could also affect NK cells expressing the NKG2D-based CAR, interaction of sMICA with NKAR-NK-92 cells was investigated by flow cytometry. Thereby, strong binding of sMICA to the surface of the CAR-NK cells was found, which was blocked in a concentration-dependent manner by NKAB-ErbB2 (figure 6C), indicating that the bispecific antibody can shield NKG2D. Occupation of the ligand binding site of the NKG2D-CAR by SMICA was also relevant for cytotoxic activity of NKAR-NK-92 cells, which was readily triggered by NKG2DLs naturally expressed by MDA-MB453 breast cancer cells, but markedly inhibited in the presence of competing sMICA (figure 6D). In contrast, the enhanced cell killing activity of the combination of NKAR-NK-92 cells and NKAB-ErbB2 was not significantly affected by an excess of sMICA, suggesting that this strategy could overcome immune evasion due to ligand shedding.

In vivo activity of NKAR-NK-92 cells and NKAB-ErbB2 antibody against ErbB2-positive glioblastoma tumors

To investigate the combined effect of NKAR-NK cells and NKAB-ErbB2 antibody in vivo in a setting where tumor cells similar to cancer stem cells lack NKG2DLs that could trigger the NKG2D-CAR directly, ${ }^{20}$ a subcutaneous tumor 
model based on syngeneic GL261/ErbB2 glioblastoma tumors in immunocompetent C57BL/6 mice was established. ${ }^{2733}$ Since GL261/ErbB2 cells are of murine origin, endogenous NKG2DLs expressed by these cells are not recognized by the NKAR molecule, which is based on human NKG2D. Consequently, in in vitro cytotoxicity assays no difference in sensitivity of GL261/ErbB2 cells to NKAR-NK-92 and parental NK-92 was found (figure 7A). For NK-92 cells, this remained unchanged in the presence of NKAB-ErbB2. However, when NKAR-NK-92 cells were combined with NKAB-ErbB2, even at low $\mathrm{E} / \mathrm{T}$ ratios a marked increase in cytotoxicity against GL261/ErbB2 was observed. Thereby, intact homodimeric NKAB-ErbB2 was more active against these target cells than a mutated NKAB-ErbB2 $\left(\mathrm{C}_{106} \mathrm{~S}\right.$ and $\left.\mathrm{C}_{109} \mathrm{~S}\right)$ derivative which cannot form dimers due to the lack of intermolecular disulfide bridges within the $\operatorname{IgG}_{4}$ hinge region (online supplemental figure S7). Likewise, NKAB-ErbB2-mediated recognition of murine melanoma cells genetically modified to express human ErbB2 induced cytokine secretion and specific lysis by NKAR-NK-92 cells (online supplemental figure S5).

For in vivo analysis, 7 days after tumor cell inoculation, mice were treated by peritumoral injection of $1 \times 10^{7}$ NKAR-NK-92 or parental NK-92 cells with or without $5 \mu \mathrm{g}$ of NKAB-ErbB2 antibody admixed to the injection medium twice per week for 3 weeks. Thereby, combination treatment with the NKAR-NK cells and NKAB-ErbB2 was highly effective. Tumor outgrowth was controlled in eight out of nine animals in this group during therapy, and complete tumor regression was seen in seven of the mice thereafter, leaving no measurable tumors 3 months after the last treatment at termination of the experiment on day 115 (figure 7B). Since the NKAR receptor cannot recognize GL261/ErbB2 cells on its own, treatment with NKAR-NK-92 cells alone had no effect on tumor development during or after therapy, with only one out of eight mice in this group being tumor-free at endpoint analysis. This was most likely due to spontaneous rejection not related to the treatment. ${ }^{27}$ While combination therapy with parental NK-92 cells and the NKAB-ErbB2 antibody showed some effect and resulted in delayed tumor growth, still only two of nine animals in this group presented as tumor-free on day 115 (figure 7B). The different kinetics in tumor growth among the treatment groups were also reflected in symptom-free survival. While mice in the NKAR-NK-92 only group had to be sacrificed due to disease progression earlier than in the group receiving the NK-92/NKAB-ErbB2 combination (median survival of 36.5 vs 46.0 days), this difference was not statistically significant (figure 7C). In contrast, with seven animals surviving and only two mice showing delayed tumor development, median survival in the NKAR-NK-92/NKABErbB2 combination group was not reached ( $>115$ days).

Sera from animals of this group contained IgG antibodies strongly reactive with GL261/ErbB2 as well as ErbB2-negative parental GL261 glioblastoma cells (figure 7D), suggesting that an endogenous antitumor immune response induced by the NKAR-NK-92/NKABErbB2 combination treatment likely contributed to tumor rejection. To evaluate that further, the expression of PD-1 by T cells from two tumor-bearing animals of each treatment group was compared. While in comparison to splenic $\mathrm{T}$ cells and $\mathrm{T}$ cells from peripheral blood higher PD-1 expression was found for tumor-infiltrating CD $4^{+}$ and $\mathrm{CD} 8^{+} \mathrm{T}$ cells in all three treatment groups, especially for $\mathrm{CD}^{+} \mathrm{T}$ cells this was much more pronounced in mice after NKAR-NK-92/NKAB-ErbB2 combination therapy (figure 7E), which is indicative of a higher proportion of previously activated and antigen-experienced $\mathrm{T}$ cells in their tumor tissues.

\section{DISCUSSION}

Activation of endogenous NKG2D and NKG2D-based CARs can be inhibited or prevented by shedding and release of soluble NKG2DLs or downregulation of NKG2DL expression, which can contribute to immune escape. ${ }^{20} 23$ To circumvent this problem but still employ activation of NKG2D as a potent mechanism for the elimination of cancer cells, here we developed a bispecific antibody which simultaneously recognizes NKG2D and the tumor-associated antigen ErbB2, resulting in targeted activation of NKG2D and NKG2D-CAR expressing effectors against ErbB2-positive cancer cells.

Recombinant NKAB-ErbB2 antibody displayed specific binding to NKG2D and ErbB2, thereby crosslinking lymphocytes and tumor cells. The bispecific molecule improved cytotoxicity of donor-derived bulk PBMCs and ex vivo expanded primary NK cells endogenously expressing NKG2D and markedly enhanced cell killing activity of NK cells carrying an NKG2D-based CAR (NKAR) against otherwise NK-resistant ErbB2-positive targets. This included JIMT-1 breast cancer cells, which express NKG2DLs and elevated levels of ErbB2 (online supplemental figure S1) but are resistant to the clinically approved ErbB2-targeted therapeutics trastuzumab and lapatinib. ${ }^{35}$ Only picomolar concentrations of NKAB-ErbB2 were needed for maximum activity against ErbB2-expressing target cells in titration experiments with effector lymphocytes carrying natural NKG2D or transduced with the NKAR vector. Similar results were obtained when NKAB-ErbB2 was combined with T cells harboring an NKG2D-CAR (online supplemental figure S6). Importantly, while sMICA used as a model for a shed NKG2DL blocked activation of the NKAR molecule by endogenous NKG2DLs on the tumor cell surface, even a 100-fold excess of sMICA did not significantly affect the combined activity of NKAR and NKAB-ErbB2. This suggests that also in a clinical situation NKAB-ErbB2 may overcome the detrimental effects of ligand shedding on NKG2D-dependent immune surveillance. ${ }^{23}$

Activation by natural NKG2DLs or NKAB-ErbB2 towards tumor cells was much more pronounced for NKAR-expressing effector cells than lymphocytes that only harbored endogenous NKG2D, which, in 

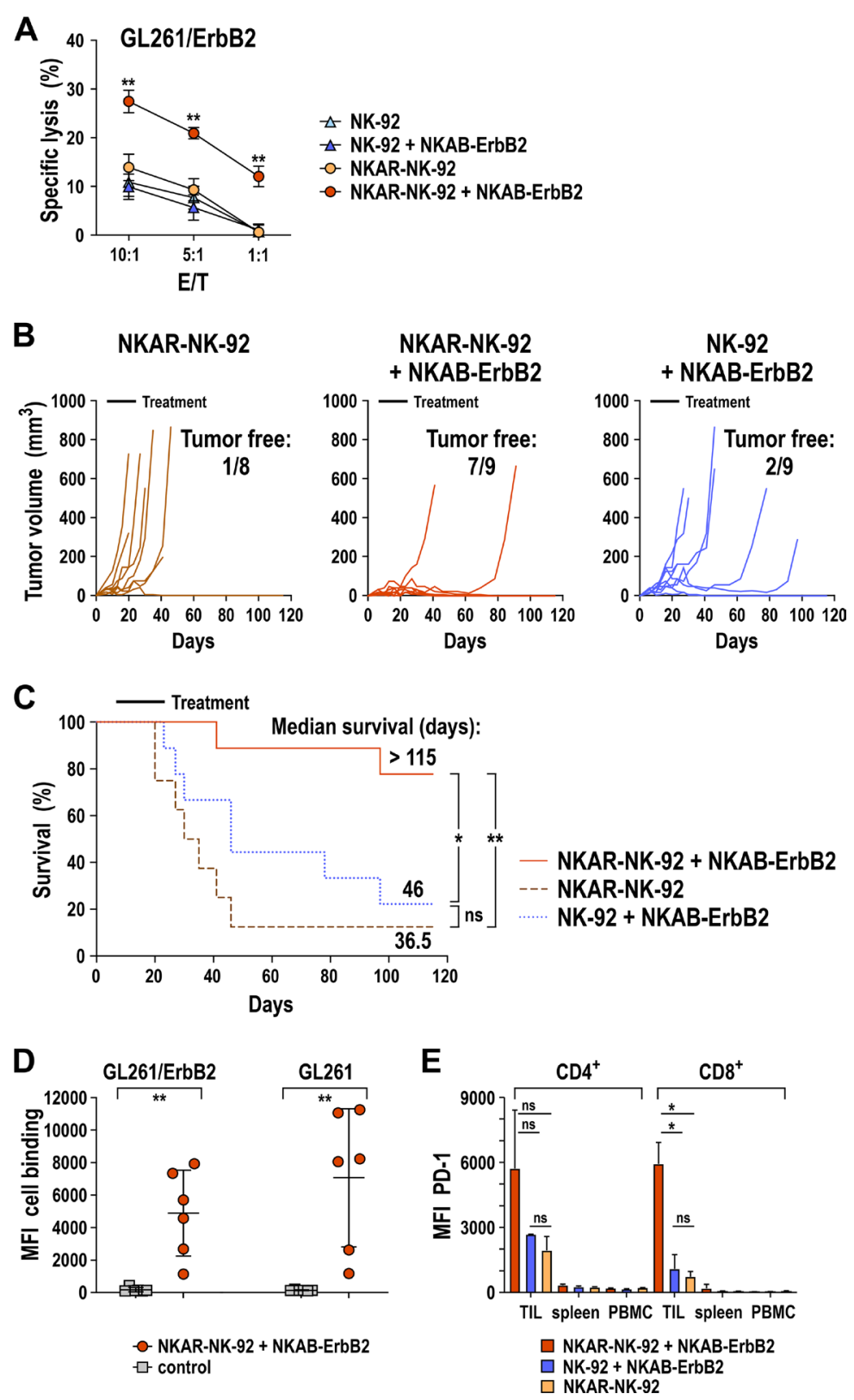

Figure 7 Combined in vivo antitumor activity of NKAR-NK-92 cells and NKAB-ErbB2 antibody against syngeneic glioblastoma in immunocompetent C57BL/6 mice. (A) Cytotoxicity of NKAR-NK-92 (orange circles) and NK-92 cells (light blue triangles) in the absence, and NKAR-NK-92 (red circles) and NK-92 cells (dark blue triangles) in the presence of $0.16 \mathrm{nM}(25 \mathrm{ng} / \mathrm{mL}$ ) of NKABErbB2 against murine GL261/ErbB2 glioblastoma cells expressing human ErbB2 was investigated in flow cytometry-based cytotoxicity assays after coincubation at different $E / T$ ratios for 3 hours. Mean values $\pm S D$ are shown; $n=3$ technical replicates from a representative experiment. Data were analyzed by two-tailed unpaired Student's t-test (shown for NKAR-NK-92+NKABErbB2 vs NKAR-NK-92). ${ }^{* *} P<0.01$. (B) GL261/ErbB2 cells $\left(1 \times 10^{6}\right)$ were subcutaneously injected into the right flank of C57BL/6 mice. Seven days later, the mice were treated by peritumoral injection of $1 \times 10^{7}$ NKAR-NK-92 cells without $(n=8)$ or with $5 \mu \mathrm{g}$ of NKAB-ErbB2 antibody $(n=9)$ admixed to the cells two times per week for 3 weeks. Control mice received parental NK-92 cells with NKAB-ErbB2 $(n=9)$. Tumor growth in the individual animals was followed by caliper measurements. (C) Symptom-free survival of the mice. Data were analyzed by Kaplan-Meier plot and log-rank test. ${ }^{*} P<0.05,{ }^{* *} P<0.01$. ns: $p>0.05$. (D) Induction of antibodies against glioblastoma cells in animals from the experiment shown in (B) and (C) treated with NKAR-NK-92 and NKABErbB2 $(n=6)$ was investigated by determining cell surface binding of IgG from serum to GL261/ErbB2 and ErbB2-negative parental GL261 cells as indicated (red circles) by flow cytometry. Sera from naïve mice $(n=6)$ served as controls (gray squares). MFI: geometric mean. Mean values $\pm S D$ are shown. Data were analyzed by two-tailed unpaired Student's t-test. ${ }^{*} P<0.01$. (E) PD-1 expression by $\mathrm{CD}^{+}$and $\mathrm{CD}^{+}{ }^{+} \mathrm{T}$ cells from TILs, splenocytes (spleen) and PBMCs of animals from the experiment (B,C) treated with NKAR-NK-92 (orange bars, $n=2$ ), NKAR-NK-92 and NKAB-ErbB2 (red bars, $n=2$ ), or NK-92 and NKAB-ErbB2 (blue bars, $n=2$ ) was determined by flow cytometry with anti-PD-1 antibody. Cells were gated using anti-CD4 and anti-CD8 antibodies as depicted in online supplemental figure $S 8$. Mean values \pm SD are shown. Data were analyzed by two-tailed unpaired Student's t-test (shown for TILs). ${ }^{*} \mathrm{P}<0.05$. ns: $\mathrm{p}>0.05$. MFI, mean fluorescence intensity; ns, not significant; PBMC, peripheral blood mononuclear cell; PD-1, programmed cell death protein 1; TIL, tumor-infiltrating lymphocyte. 
addition to different expression levels of NKG2D and the NKG2D-CAR, was likely due to distinct signaling capabilities of the receptors. To be active, human NKG2D requires prior association with the adaptor molecule DNAXactivating protein of $10 \mathrm{kDa}$ (DAP10).$^{36}$ In contrast, in the NKG2D-CAR employed in our study, extracellular target recognition and intracellular signaling functions are directly linked in one molecule, likely accelerating kinetics of effector cell activation. In addition, DAP10 only contains a single YXXM tyrosine-based motif similar to the ones found in the costimulatory molecules CD28 and ICOS for interaction with downstream pathways. ${ }^{37}$ Instead, the NKG2D-CAR harbors CD3ל, which contributes three potent immunoreceptor tyrosine-based activation motifs. ${ }^{38}$ On their own or in combination, ectopic expression of the NKG2D-CAR and application of the bispecific NKAB molecule may also bypass reduced responsiveness and downregulation of endogenous NKG2D observed on chronic stimulation by NKG2DLs and during NK cell exhaustion. ${ }^{39-41}$

The bispecific NKAB-ErbB2 antibody mimics the general structure of a human IgG molecule, with the hinge, $\mathrm{CH} 2$ and $\mathrm{CH} 3$ domains of the $\mathrm{IgG}_{4} \mathrm{Fc}$ region separating NKG2D-specific and ErbB2-specific antibody domains. Accordingly, in contrast to other bispecific molecules with only one binding site for NKG2D and a target antigen, ${ }^{42-44}$ the NKAB-ErbB2 protein was expressed as a homodimer with two binding sites each for NKG2D and ErbB2. For comparison, we also generated a mutated NKAB-ErbB2 $\left(\mathrm{C}_{106} \mathrm{~S}\right.$ and $\left.\mathrm{C}_{109} \mathrm{~S}\right)$ derivative which lacked the cysteine residues within the $\operatorname{IgG}_{4}$ hinge region and was therefore only produced as a monomer (online supplemental figure S7). In short-term assays, dimeric and monomeric NKAB-ErbB2 proteins similarly enhanced cytotoxicity of NKG2D-CAR NK cells against target cells coexpressing NKG2DLs and ErbB2. However, when target recognition was entirely dependent on NKABErbB2 due to the lack of endogenous ligands for human NKG2D, homodimeric NKAB-ErbB2 was more effective, suggesting that the tetravalent design may be of particular advantage in a setting of escape from natural NKG2D immune surveillance (online supplemental figure S7). Furthermore, with around $180 \mathrm{kDa}$, the apparent molecular mass of intact NKAB-ErbB2 is close to that of regular IgG molecules, which similar to complete antibodies will likely translate to a longer in vivo half-life when compared with monomeric scFv-scFv or scFv-Fc molecules. ${ }^{45}$

We based the NKAB-ErbB2 molecule on the structure of $\mathrm{IgG}_{4}$, which does not bind to CD16 with high affinity, allowing to distinguish NKG2D- from CD16mediated effects. This NKAB design proved highly flexible, allowing to switch the positions of NKG2D- and ErbB2-specific binding domains within the molecule without loss of activity (online supplemental figure S4), indicating that the overall $\mathrm{NKAB}$ structure will be applicable also for similar bispecific molecules targeting alternative tumor antigens and/or activating lymphocyte receptors. ${ }^{46}$ Likewise, exchanging the $\mathrm{IgG}_{4} \mathrm{Fc}$ region with the respective sequence of $\operatorname{IgG}_{1}$ led to a fully functional molecule, although without a clear benefit of the possible simultaneous or alternative interaction of the resulting NKAB-ErbB2 $\left(\operatorname{IgG}_{1}\right)$ antibody with NKG2D and CD16 (online supplemental figure S3). Nevertheless, engineering of the $\operatorname{IgG}_{1} \mathrm{Fc}_{\mathrm{c}}$ domain to include mutations that enhance affinity for CD16 may still increase activity of such $\mathrm{IgG}_{1}$-based NKAB molecules. ${ }^{47}$

In vivo antitumor activity of NKAB-ErbB2 in combination with NKAR-NK-92 cells was investigated in a murine glioblastoma model. In this disease, activity of the NKG2D/NKG2DL system can be negatively affected by limited expression of NKG2DLs. ${ }^{48} 49$ To mimic the situation of low or absent NKG2DL expression as a mechanism of immune evasion, we used murine GL261/ErbB2 glioblastoma cells growing as subcutaneous tumors in C57BL/6 mice. ${ }^{27}$ While GL261 cells express moderate levels of murine NKG2DLs, ${ }^{48}$ these are not recognized by human NKG2D or the human NKG2D-CAR as exemplified by the results from our in vitro cytotoxicity assays with NK-92 and NKAR-NK-92 cells. Hence, as expected, repeated treatment of established GL261/ErbB2 tumors with NKAR-NK-92 cells alone was ineffective, with rapid tumor growth continuing in most animals while still under therapy. While not resulting in a statistically significant survival benefit in comparison to NKARNK-92 monotherapy, tumor outgrowth was delayed in mice treated with a combination of NKAB-ErbB2 and parental NK-92 cells, where the bispecific antibody can act via endogenous NKG2D expressed at moderate levels. However, when NKAR-NK-92 cells were applied together with NKAB-ErbB2, the glioblastoma tumors were readily rejected in the majority of the animals, resulting in cures with seven out of nine mice in this group staying tumorfree during the remaining course of the experiment.

In addition to direct tumor cell lysis, activated NK cells play a critical role in dendritic cell (DC) recruitment and maturation, thus indirectly inducing or enhancing tumor-specific adaptive immune responses. ${ }^{50}$ This likely contributed to the durable responses observed in immunocompetent mice after treatment with the NKAB-ErbB2/ NKAR-NK-92 combination, as seen in previous studies with NK-92 cells which express a more classical scFv-based CAR and are currently evaluated in a phase I clinical trial in patients with glioblastoma. ${ }^{27} 51$ On NKAB-ErbB2mediated activation, NKAR-NK-92 cells markedly upregulated the secretion of proinflammatory chemokines and cytokines pivotal in DC recruitment (online supplemental figure S5), including RANTES (CCL5), ${ }^{52}$ MIP-1 $\alpha$ (CCL3) and MIP-1 $\beta$ (CCL4), ${ }^{53}$ or DC maturation and function, such as GM-CSF, ${ }^{54}$ IFN- $\gamma$, TNF- $\alpha$ and TNF- $\beta .^{50}{ }^{55}$ Indeed, sera from mice cured of their glioblastoma tumors after combination therapy with NKAB-ErbB2 and NKARNK-92 contained IgG antibodies reactive with GL261 and GL261/ErbB2 cells. While we did not investigate whether combined treatment with NKAB-ErbB2 and NKAR-NK-92 also resulted in the activation of tumor-reactive $\mathrm{T}$ cells, TILs from the two animals in this group that were not 
cured expressed higher levels of the immune checkpoint molecule PD-1 than TILs from mice treated with NKARNK-92 alone or a combination of NKAB-ErbB2 with parental NK-92 cells. Despite the failure in these mice to reject the tumors, this is indicative of more effective TCR-mediated stimulation of tumor-infiltrating T cells on NKAB-ErbB2/NKAR-NK-92 therapy ${ }^{56}$ Importantly, these data also suggest that combining NKAB-ErbB2 further with an immune checkpoint inhibitor may be warranted.

\section{CONCLUSION}

Taken together, our data show that the bispecific NKAB antibody efficiently redirects effector lymphocytes to cancer cells expressing the respective tumor-associated antigen, thereby overcoming the inhibitory effects of low expression or shedding of NKG2DLs and resulting in enhanced antitumor activity and therapy-induced adaptive antitumor immune responses. In particular, the combination of an NKAB antibody with NK or T cells engineered with an NKG2D-based chimeric antigen receptor represents a versatile approach to simultaneously enhance tumor-antigen-specific and natural NKG2D-mediated cytotoxicity, which may become very useful to target tumors with heterogeneous target antigen expression not amenable to classical CAR-T or CAR-NK cell therapies.

Acknowledgements The authors thank Stephen Gottschalk for advice on T-cell transduction, Stefan Stein and Annette Trzmiel for flow cytometric cell sorting, Barbara Uherek and Thorsten Geyer for technical assistance, Tijna Alekseeva for advice on time-lapse imaging and the staff at the animal facility of the GeorgSpeyer-Haus for their support.

Contributors $\mathrm{CZ}, \mathrm{PO}$ and WSW designed the study. CZ, PO, JR, AS, MB, JPS, AB, $A W$ and NM performed the experiments and acquired data. $C Z, P O, J R, A S, M B, J P S$, $A B$ and WSW analyzed data. CZ and WSW wrote the manuscript. All authors were involved in the critical review and editing of the manuscript and approved the final version.

Funding This work was supported in part by grants from the German Cancer Consortium, the Deutsche Forschungsgemeinschaft (WE 2589/4-1 and WE 2589/6-1), the LOEWE Center Frankfurt Cancer Institute (HMWK III L 5-519/03/03.001-0015), and institutional funds of Georg-Speyer-Haus. GeorgSpeyer-Haus is funded jointly by the German Federal Ministry of Health and the Hessen State Ministry of Higher Education, Research and the Arts.

Competing interests $\mathrm{CZ}, \mathrm{PO}, \mathrm{AS}$, AW and WSW are named as inventors on patents and patent applications in the field of cancer immunotherapy owned by GeorgSpeyer-Haus.

\section{Patient consent for publication Not applicable.}

Provenance and peer review Not commissioned; externally peer reviewed.

Data availability statement All data relevant to the study are included in the article or uploaded as supplementary information.

Supplemental material This content has been supplied by the author(s). It has not been vetted by BMJ Publishing Group Limited (BMJ) and may not have been peer-reviewed. Any opinions or recommendations discussed are solely those of the author(s) and are not endorsed by BMJ. BMJ disclaims all liability and responsibility arising from any reliance placed on the content. Where the content includes any translated material, BMJ does not warrant the accuracy and reliability of the translations (including but not limited to local regulations, clinical guidelines, terminology, drug names and drug dosages), and is not responsible for any error and/or omissions arising from translation and adaptation or otherwise.

Open access This is an open access article distributed in accordance with the Creative Commons Attribution Non Commercial (CC BY-NC 4.0) license, which permits others to distribute, remix, adapt, build upon this work non-commercially, and license their derivative works on different terms, provided the original work is properly cited, appropriate credit is given, any changes made indicated, and the use is non-commercial. See http://creativecommons.org/licenses/by-nc/4.0/.

\section{ORCID iD}

Winfried S Wels http://orcid.org/0000-0001-9858-3643

\section{REFERENCES}

1 June $\mathrm{CH}$, O'Connor RS, Kawalekar OU, et al. CAR T cell immunotherapy for human cancer. Science 2018;359:1361-5.

2 Gad AZ, El-Naggar S, Ahmed N. Realism and pragmatism in developing an effective chimeric antigen receptor T-cell product for solid cancers. Cytotherapy 2016;18:1382-92.

3 DeRenzo C, Krenciute G, Gottschalk S. The landscape of CAR T cells beyond acute lymphoblastic leukemia for pediatric solid tumors. Am Soc Clin Oncol Educ Book 2018;11:830-7.

4 Sotillo E, Barrett DM, Black KL, et al. Convergence of acquired mutations and alternative splicing of CD19 enables resistance to CART-19 immunotherapy. Cancer Discov 2015;5:1282-95.

5 Genßler S, Burger MC, Zhang C, et al. Dual targeting of glioblastoma with chimeric antigen receptor-engineered natural killer cells overcomes heterogeneity of target antigen expression and enhances antitumor activity and survival. Oncoimmunology 2016;5:e1119354.

6 O'Rourke DM, Nasrallah MP, Desai A, et al. A single dose of peripherally infused EGFRvlll-directed CAR T cells mediates antigen loss and induces adaptive resistance in patients with recurrent glioblastoma. Sci Trans/ Med 2017;9:eaaa0984.

7 Ruella M, Barrett DM, Kenderian SS, et al. Dual CD19 and CD123 targeting prevents antigen-loss relapses after CD19-directed immunotherapies. J Clin Invest 2016;126:3814-26.

8 Grada Z, Hegde M, Byrd T, et al. TanCAR: a novel bispecific chimeric antigen receptor for cancer immunotherapy. Mol Ther Nucleic Acids 2013;2:e105.

9 Zhang T, Lemoi BA, Sentman CL. Chimeric NK-receptor-bearing T cells mediate antitumor immunotherapy. Blood 2005;106:1544-51.

10 Spear P, Wu M-R, Sentman M-L, et al. NKG2D ligands as therapeutic targets. Cancer Immun 2013;13:8.

11 Lazarova M, Wels WS, Steinle A. Arming cytotoxic lymphocytes for cancer immunotherapy by means of the NKG2D/NKG2D-ligand system. Expert Opin Biol Ther 2020;20:1491-501.

12 Raulet DH, Gasser S, Gowen BG, et al. Regulation of ligands for the NKG2D activating receptor. Annu Rev Immunol 2013;31:413-41.

13 Lanier LL. NKG2D receptor and its ligands in host defense. Cancer Immunol Res 2015;3:575-82.

14 Strong RK. Asymmetric ligand recognition by the activating natural killer cell receptor NKG2D, a symmetric homodimer. Mol Immunol 2002;38:1029-37.

15 Nausch N, Cerwenka A. NKG2D ligands in tumor immunity. Oncogene 2008;27:5944-58.

16 Ullrich E, Koch J, Cerwenka A, et al. New prospects on the NKG2D/ NKG2DL system for oncology. Oncoimmunology 2013;2:e26097.

17 Le Bert N, Gasser S. Advances in NKG2D ligand recognition and responses by NK cells. Immunol Cell Biol 2014;92:230-6.

18 Chang Y-H, Connolly J, Shimasaki N, et al. A chimeric receptor with NKG2D specificity enhances natural killer cell activation and killing of tumor cells. Cancer Res 2013;73:1777-86.

19 Baumeister SH, Murad J, Werner L, et al. Phase I trial of autologous CAR T cells targeting NKG2D ligands in patients with AML/MDS and multiple myeloma. Cancer Immunol Res 2019;7:100-12.

20 Paczulla AM, Rothfelder K, Raffel S, et al. Absence of NKG2D ligands defines leukaemia stem cells and mediates their immune evasion. Nature 2019;572:254-9.

21 Schwinn N, Vokhminova D, Sucker A, et al. Interferon- $\gamma$ downregulates NKG2D ligand expression and impairs the NKG2Dmediated cytolysis of MHC class I-deficient melanoma by natural killer cells. Int J Cancer 2009;124:1594-604.

22 Eisele G, Wischhusen J, Mittelbronn M, et al. TGF-Beta and metalloproteinases differentially suppress NKG2D ligand surface expression on malignant glioma cells. Brain 2006;129:2416-25.

23 Salih HR, Rammensee H-G, Steinle A. Cutting edge: down-regulation of MICA on human tumors by proteolytic shedding. J Immunol 2002;169:4098-102.

24 Groh V, Wu J, Yee C, et al. Tumour-derived soluble MIC ligands impair expression of NKG2D and T-cell activation. Nature 2002;419:734-8.

25 Groth A, Klöss S, Pogge von Strandmann E, et al. Mechanisms of tumor and viral immune escape from natural killer cell-mediated surveillance. J Innate Immun 2011;3:344-54. 
26 Xu Y, Darcy PK, Kershaw MH. Tumor-specific dendritic cells generated by genetic redirection of Toll-like receptor signaling against the tumor-associated antigen, ErbB2. Cancer Gene Ther 2007:14:773-80.

27 Zhang C, Burger MC, Jennewein L, et al. ErbB2/HER2-specific NK cells for targeted therapy of glioblastoma. J Natl Cancer Inst 2016;108:djv375

28 Kwong KY, Baskar S, Zhang H, et al. Generation, affinity maturation, and characterization of a human anti-human NKG2D monoclonal antibody with dual antagonistic and agonistic activity. J Mol Biol 2008;384:1143-56.

29 Wels W, Harwerth I-M, Zwickl M, et al. Construction, bacterial expression and characterization of a bifunctional single-chain antibody-phosphatase fusion protein targeted to the human ERBB-2 receptor. Nat Biotechnol 1992;10:1128-32.

30 Schönfeld K, Sahm C, Zhang C, et al. Selective inhibition of tumor growth by clonal NK cells expressing an ErbB2/HER2-specific chimeric antigen receptor. Mol Ther 2015;23:330-8.

31 Zhou Q, Uhlig KM, Muth A, et al. Exclusive transduction of human $\mathrm{CD}^{+} \mathrm{T}$ cells upon systemic delivery of CD4-targeted lentiviral vectors. J Immunol 2015;195:2493-501.

32 Demaison C, Parsley K, Brouns G, et al. High-level transduction and gene expression in hematopoietic repopulating cells using a human Imunodeficiency virus type 1-based lentiviral vector containing an internal spleen focus forming virus promoter. Hum Gene Ther 2002;13:803-13.

33 Zhang $\mathrm{C}$, Oberoi $\mathrm{P}$, Oelsner $\mathrm{S}$, et al. Chimeric antigen receptorengineered NK-92 cells: an off-the-shelf cellular therapeutic for targeted elimination of cancer cells and induction of protective antitumor immunity. Front Immunol 2017;8:533.

34 Pende D, Parolini S, Pessino A, et al. Identification and molecular characterization of $\mathrm{NKp} 30$, a novel triggering receptor involved in natural cytotoxicity mediated by human natural killer cells. J Exp Med 1999:190:1505-16.

35 O'Brien NA, Browne BC, Chow L, et al. Activated phosphoinositide 3-kinase/AKT signaling confers resistance to trastuzumab but not lapatinib. Mol Cancer Ther 2010;9:1489-502.

36 Garrity D, Call ME, Feng J, et al. The activating NKG2D receptor assembles in the membrane with two signaling dimers into a hexameric structure. Proc Natl Acad Sci U S A 2005;102:7641-6.

37 Wu Jet al. An activating immunoreceptor complex formed by NKG2D and DAP10. Science 1999;285:730-2.

38 Isakov N. Immunoreceptor tyrosine-based activation motif (ITAM), a unique module linking antigen and $\mathrm{Fc}$ receptors to their signaling cascades. J Leukoc Biol 1997;61:6-16.

39 Alvarez M, Simonetta F, Baker J, et al. Regulation of murine NK cell exhaustion through the activation of the DNA damage repair pathway. JCI Insight 2019;4:e127729.
40 Judge SJ, Murphy WJ, Canter RJ. Characterizing the dysfunctional NK cell: assessing the clinical relevance of exhaustion, anergy, and senescence. Front Cell Infect Microbiol 2020;10:49.

41 Merino AM, Kim H, Miller JS, et al. Unraveling exhaustion in adaptive and conventional NK cells. J Leukoc Biol 2020;108:1361-8.

42 von Strandmann EP, Hansen HP, Reiners KS, et al. A novel bispecific protein (ULBP2-BB4) targeting the NKG2D receptor on natural killer (NK) cells and CD138 activates NK cells and has potent antitumor activity against human multiple myeloma in vitro and in vivo. Blood 2006; 107:1955-62.

43 Chan WK, Kang S, Youssef Y, et al. A CS1-NKG2D bispecific antibody collectively activates cytolytic immune cells against multiple myeloma. Cancer Immunol Res 2018;6:776-87.

44 Raynaud A, Desrumeaux K, Vidard L, et al. Anti-NKG2D single domain-based antibodies for the modulation of anti-tumor immune response. Oncoimmunology 2021;10:1854529.

45 Olafsen T, Wu AM. Antibody vectors for imaging. Semin Nucl Med 2010;40:167-81.

46 Peipp M, Derer S, Lohse S, et al. HER2-specific immunoligands engaging NKp30 or NKp80 trigger NK-cell-mediated lysis of tumor cells and enhance antibody-dependent cell-mediated cytotoxicity. Oncotarget 2015;6:32075-88.

47 Gauthier L, Morel A, Anceriz N, et al. Multifunctional natural killer cell engagers targeting NKp46 trigger protective tumor immunity. Cell 2019:177:e16:1701-13.

48 Friese MA, Platten M, Lutz SZ. MICA/NKG2D-mediated immunogene therapy of experimental gliomas. Cancer Res 2003;63:8996-9006.

49 Codo P, Weller M, Meister G, et al. MicroRNA-mediated downregulation of NKG2D ligands contributes to glioma immune escape. Oncotarget 2014;5:7651-62.

50 Van Elssen CHMJ, Oth T, Germeraad WTV, et al. Natural killer cells: the secret weapon in dendritic cell vaccination strategies. Clin Cancer Res 2014;20:1095-103.

51 Burger MC, Zhang C, Harter PN, et al. CAR-engineered NK cells for the treatment of glioblastoma: turning innate effectors into precision tools for cancer immunotherapy. Front Immunol 2019;10:2683.

52 Böttcher JP, Bonavita E, Chakravarty P, et al. NK cells stimulate recruitment of $\mathrm{CDC} 1$ into the tumor microenvironment promoting cancer immune control. Cell 2018;172:e14:1022-37.

53 Caux C, Ait-Yahia S, Chemin K, et al. Dendritic cell biology and regulation of dendritic cell trafficking by chemokines. Springer Semin Immunopathol 2000;22:345-69.

54 Shi Y, Liu CH, Roberts Al, et al. Granulocyte-macrophage colonystimulating factor (GM-CSF) and T-cell responses: what we do and don't know. Cell Res 2006;16:126-33.

55 Summers-deLuca LE, McCarthy DD, Cosovic B, et al. Expression of lymphotoxin- $\alpha \beta$ on antigen-specific T cells is required for DC function. J Exp Med 2007;204:1071-81.

56 Sharpe AH, Pauken KE. The diverse functions of the PD1 inhibitory pathway. Nat Rev Immunol 2018;18:153-67. 